\title{
Thermal Analysis and Simulation of the Superconducting Magnet for the SpinQuest Experiment at FermiLab
}

\author{
09/24/2019 \\ 2019 Workshop on Polarized Sources, Targets, and Polarimetry
}

Zulkaida Akbar

University of Virginia

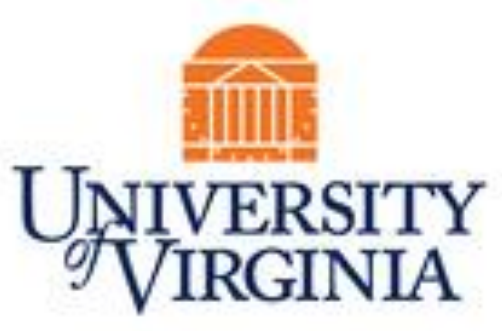

\#Fermilab 


\section{Outline}

- Introduction

- Magnetic Field Measurement \& Simulation

- Physics Processes

- Simulation Methods

- Results

- Outlook: Quench Commissioning Plan

- Backup Slide: Beam Stability Issue 


\section{Introduction: SpinQuest Experiment at Fermilab}

SpinQuest Physics:

- Sivers function for the sea quarks (main physics goal)

- Dark matter search

- Deuteron tensor function $b 1$ (longitudinal polarization)

- Gluon TMD/Twist-3 correlation function

- QCD dynamics with heavy quarks

SpinQuest as intensity frontier for the polarized-target experiment:

- $120 \mathrm{GeV}$ of proton beam

- $5 \times 10^{12}$ proton/spill. 1 spill $\approx 4.4$ seconds

- a $5 \mathrm{~T}$ of $\mathrm{Nb}$-Ti Superconducting split coil magnet

- Transversely-Polarized $\mathrm{NH}_{3}$ and $\mathrm{ND}_{3}$ targets

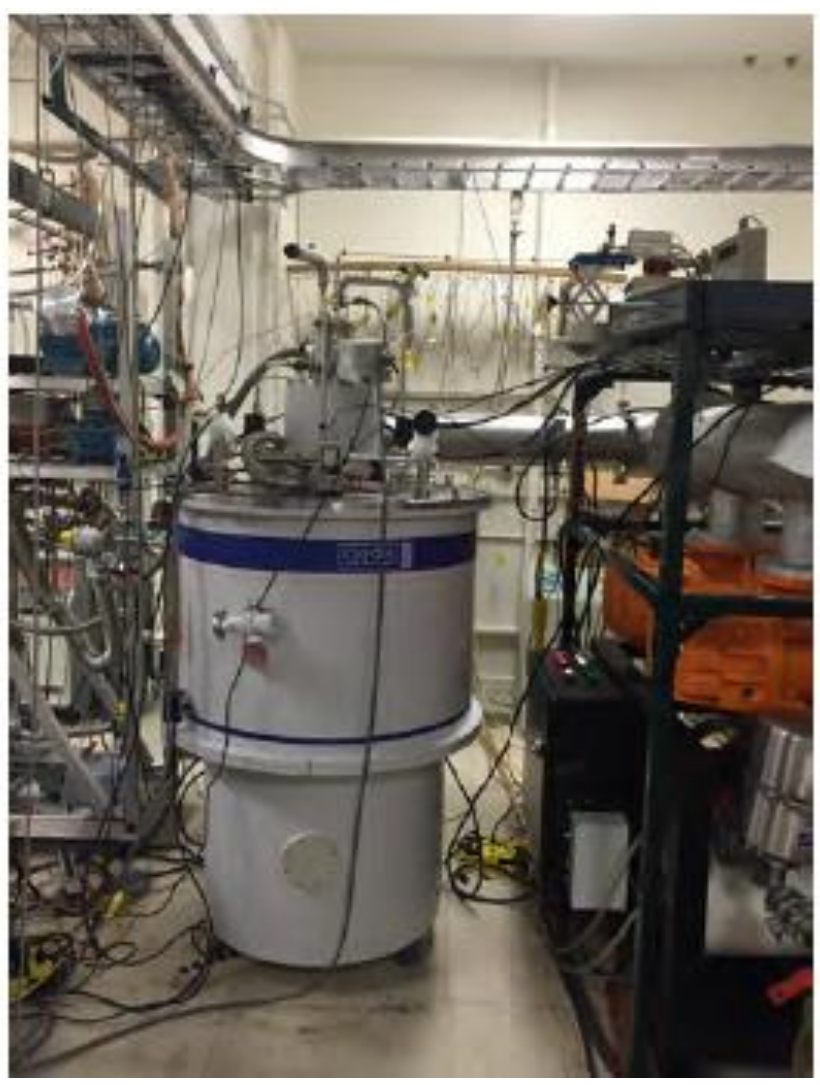

UVA/LANL Target system 


\section{Introduction: Superconducting-magnet Quench}

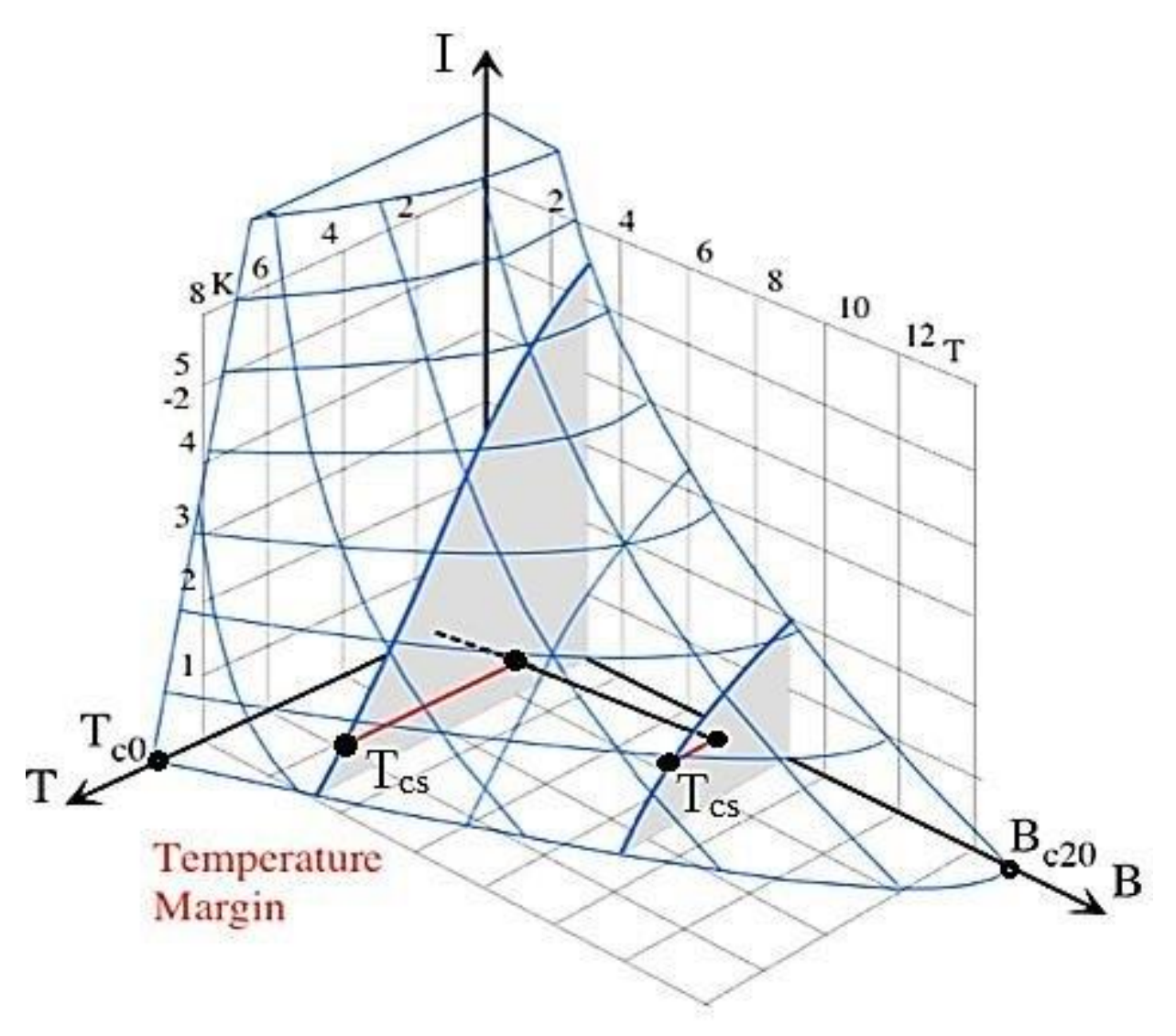

Critical surface for $\mathrm{Nb}-\mathrm{Ti}$ superconductor (courtesy of Deepak Paudel)
Critical surface for a superconductor is defined from the temperature ( $T)$, magnetic field $(B)$, and the current (I)

Magnet becomes normal conductor (quench) if the $T, B$ or I lie outside the critical surface

The magnetic field $(B)$ in the target area between the coils is $5 \mathrm{~T}$

But we do not have the information about the magnetic field in the magnet itself 


\section{Main Questions:}

- How to determine the strength of the magnetic field in the conductor?

- What is the maximum intensity of the proton beam for the SpinQuest experiment before the magnet quench? 


\section{Magnetic Field Measurement and Simulation}

\section{Motivation:}

-We need to know the magnetic field in the magnet to determine the quench limit

- But Oxford instrument only provides the magnetic field measurement inside the target cup (Along $\Delta z=7.5 \mathrm{~cm}$ and $\Delta y=3 \mathrm{~cm}$ )

- We need to measure the magnetic field outside the dewar: requires an extrapolation method into the region inside the dewar and outside the target region

- Goal: A complete 3D picture of the magnetic field insideloutside the magnet dewar

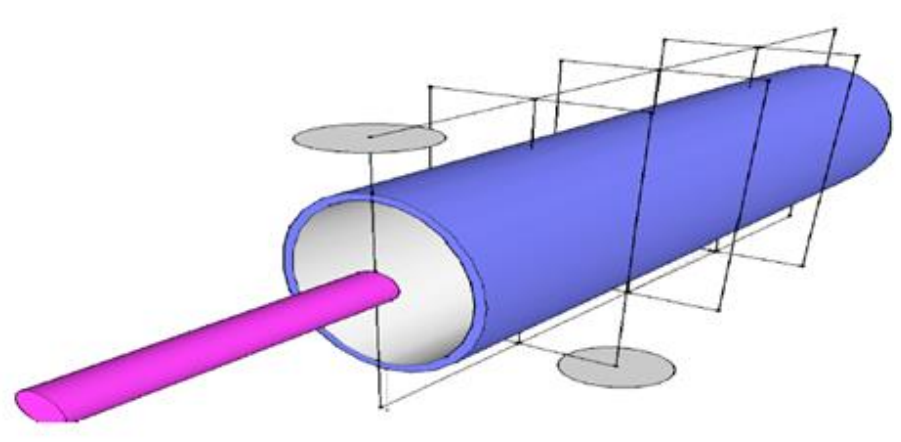

Oxford's measurements in the target area using NMR probe 


\section{Magnetic Field Measurement and Simulation}

Measurement outside the dewar during the cooldown at UVA:

Over 300 points measurement

Measure the radial and vertical component of the field

Covering 60 inch distance from the surface

Covering 5 horizontal plane and 4 different azimuthal angle

Lakeshore Gaussmeter

(Uncertainty: 20mT) 


\section{Magnetic Field Measurement and Simulation}

Challenge: There is no trivial way to fit and extrapolate the data to obtain the Magnetic field inside the dewar

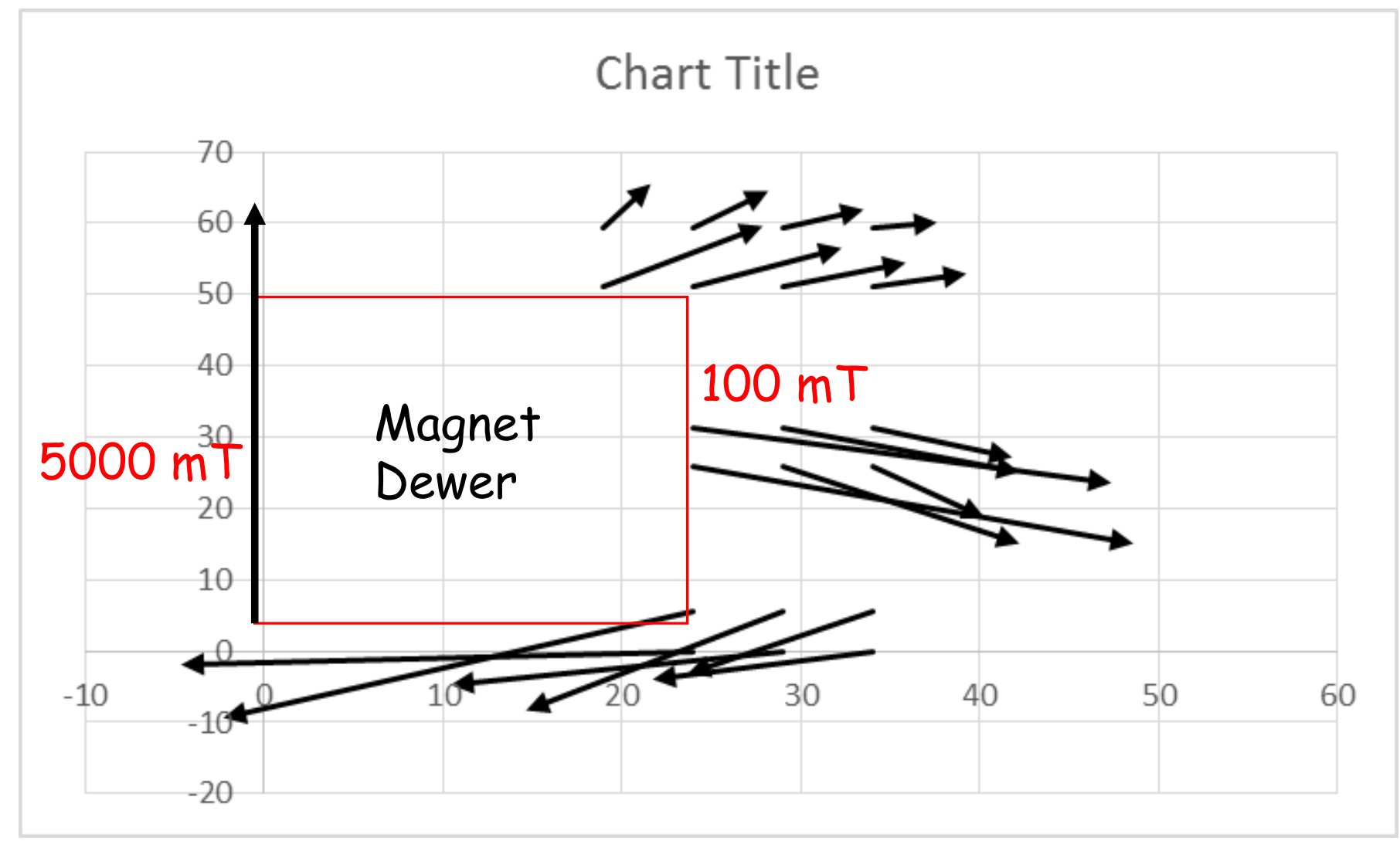

Sample of the measurements 


\section{Magnetic Field Measurement and Simulation}

Two options to address this challenge:

First, solving a set of Maxwell equation with a very complicated boundary conditions. This technique is applied by astrophysicist to extrapolate the magnetic field in solar corona from the photosphere.

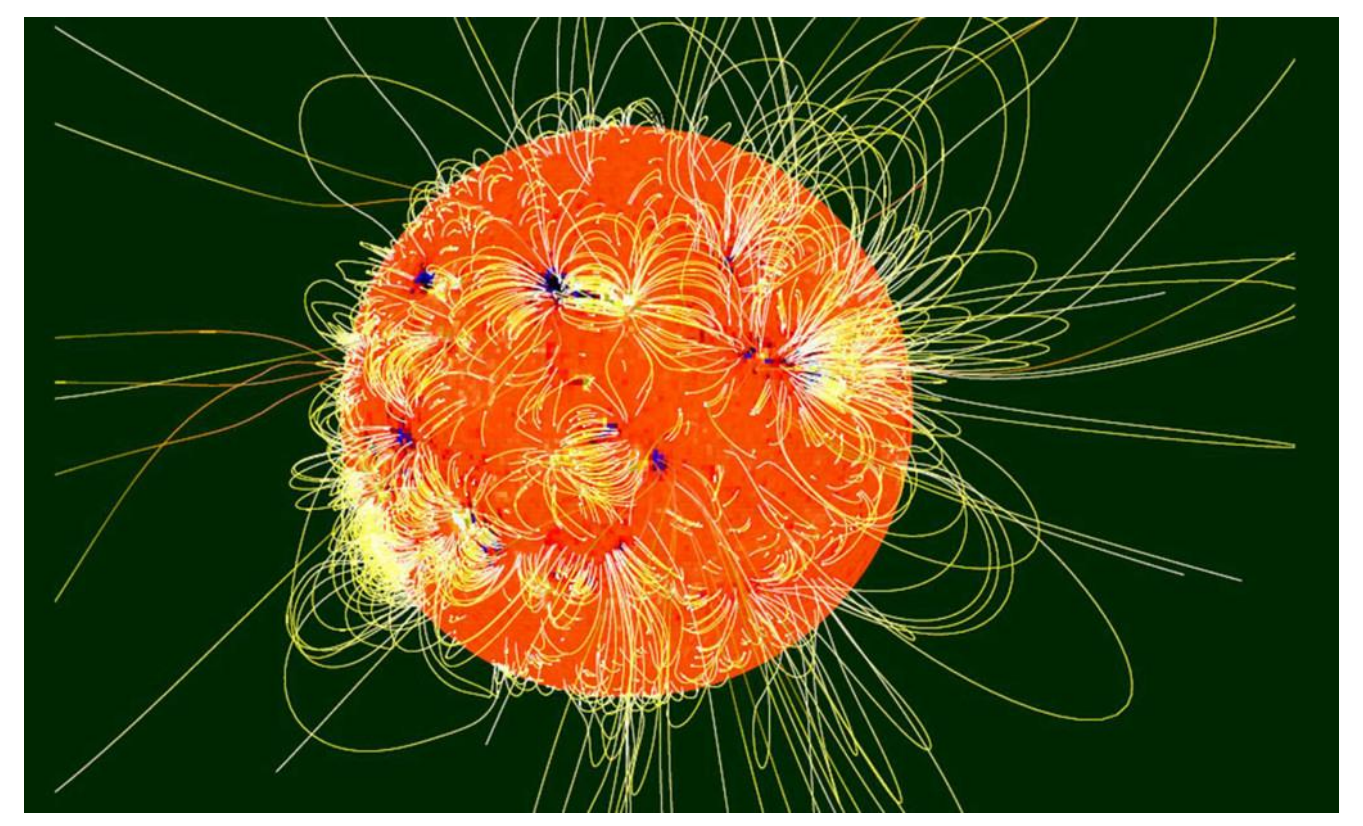

Second, using COMSOL Multiphysics to simulate the Magnet coil

Solar corona magnetic field 


\section{Magnetic Field Measurement and Simulation}

Input:

- B measurement inside the target cup

- B measurement outside the dewar

\section{Process:}

Magnetic field simulation of the superconducting coil using Finite Element Methods \& COMSOL multiphysics software

\section{Outcome:}

Put the B from the simulation into the Monte Carlo simulation

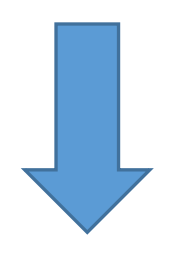

\section{Output:}

Simulation that matched the measurement results inside the target cup and outside the dewer

Notes: It is better to use the simulation results since the measurement outside the dewer use the hand probe gaussmeter which is not really accurate (the uncertainty is $20 \mathrm{mT}$ ) 


\section{Magnetic Field Measurement and Simulation}

Simulation result: we achieve a high level of homogeneity around the target area \& along the beam line:

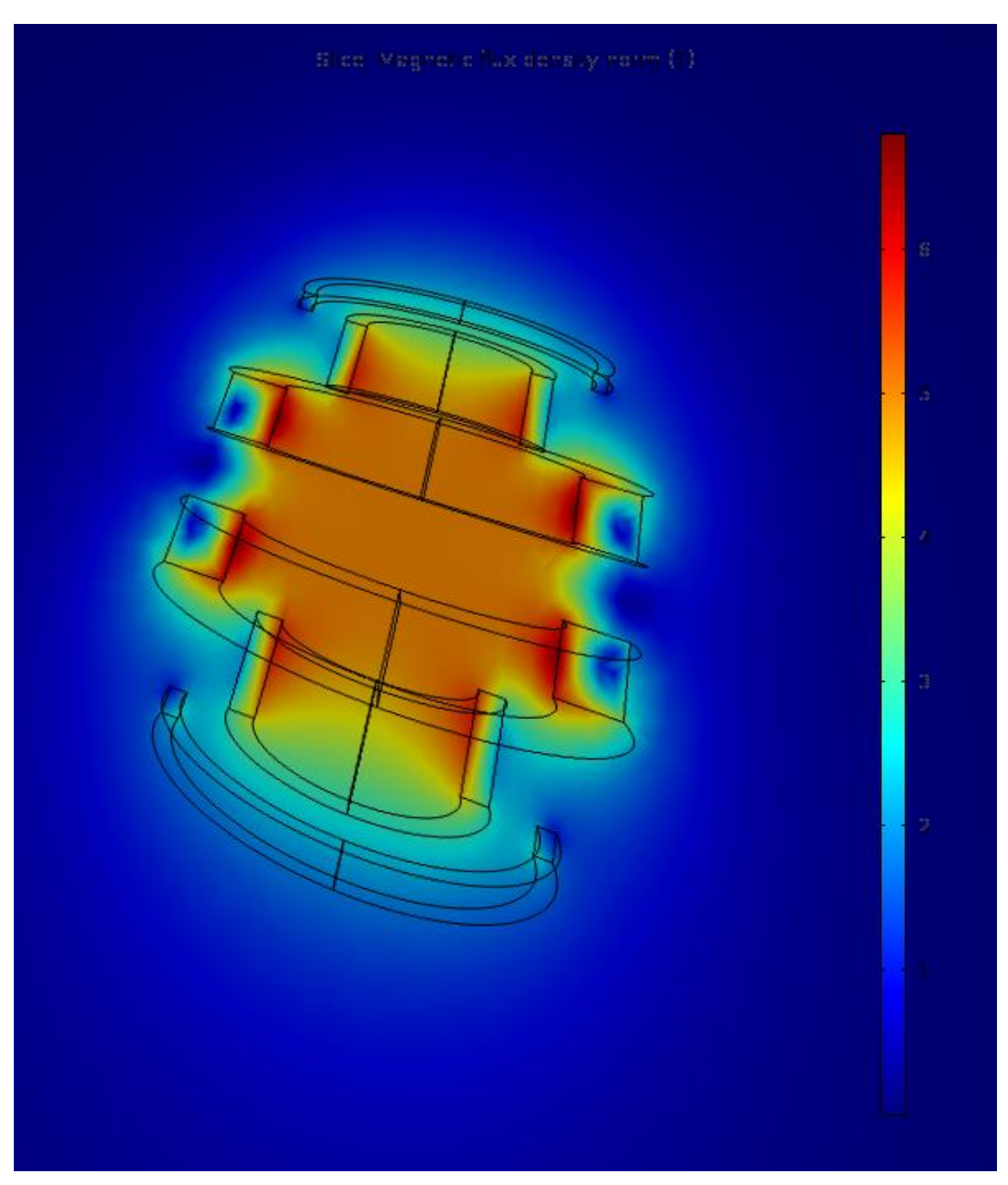

High level of homogeneity in the target area

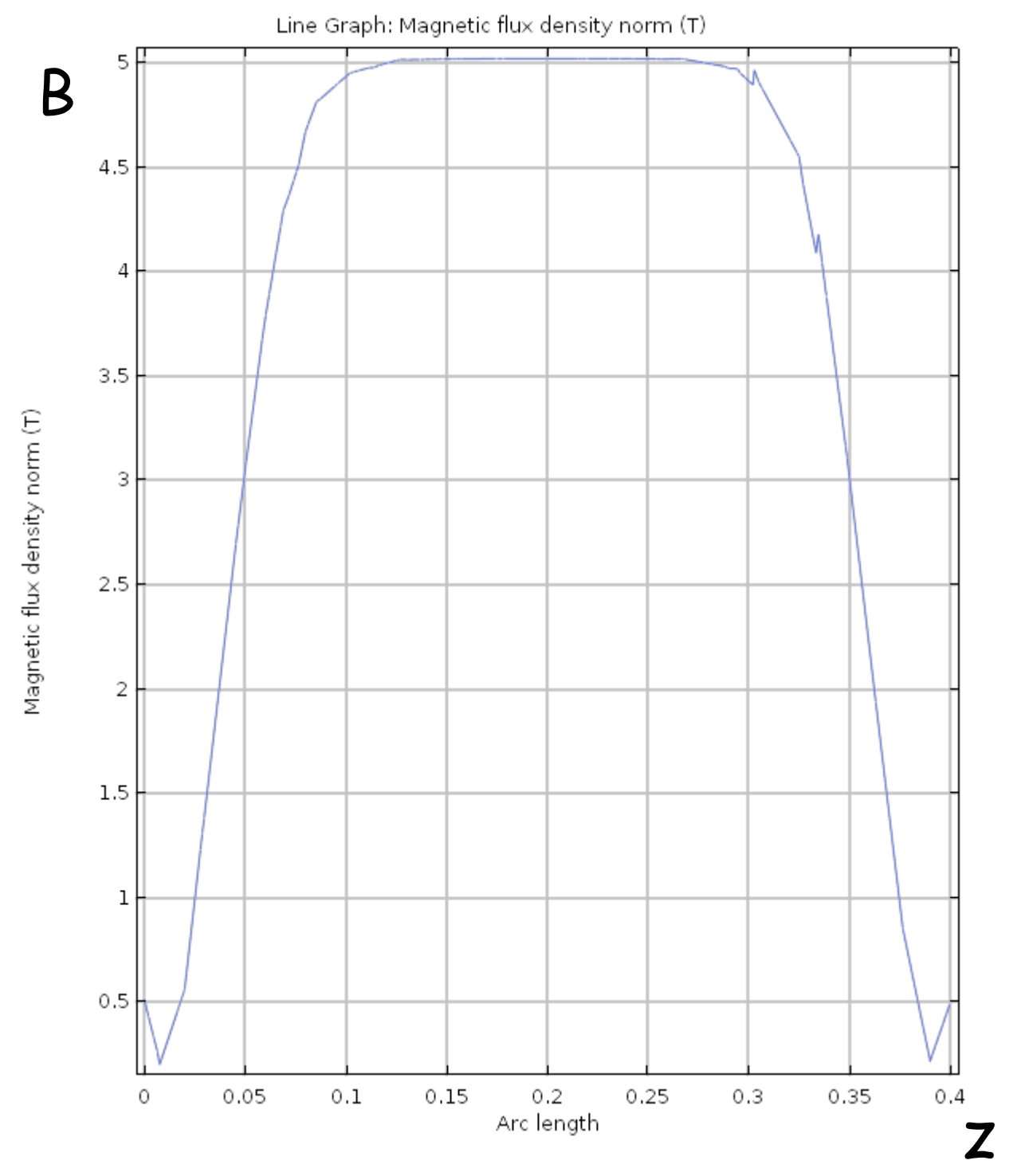




\section{And if we zoom in:}

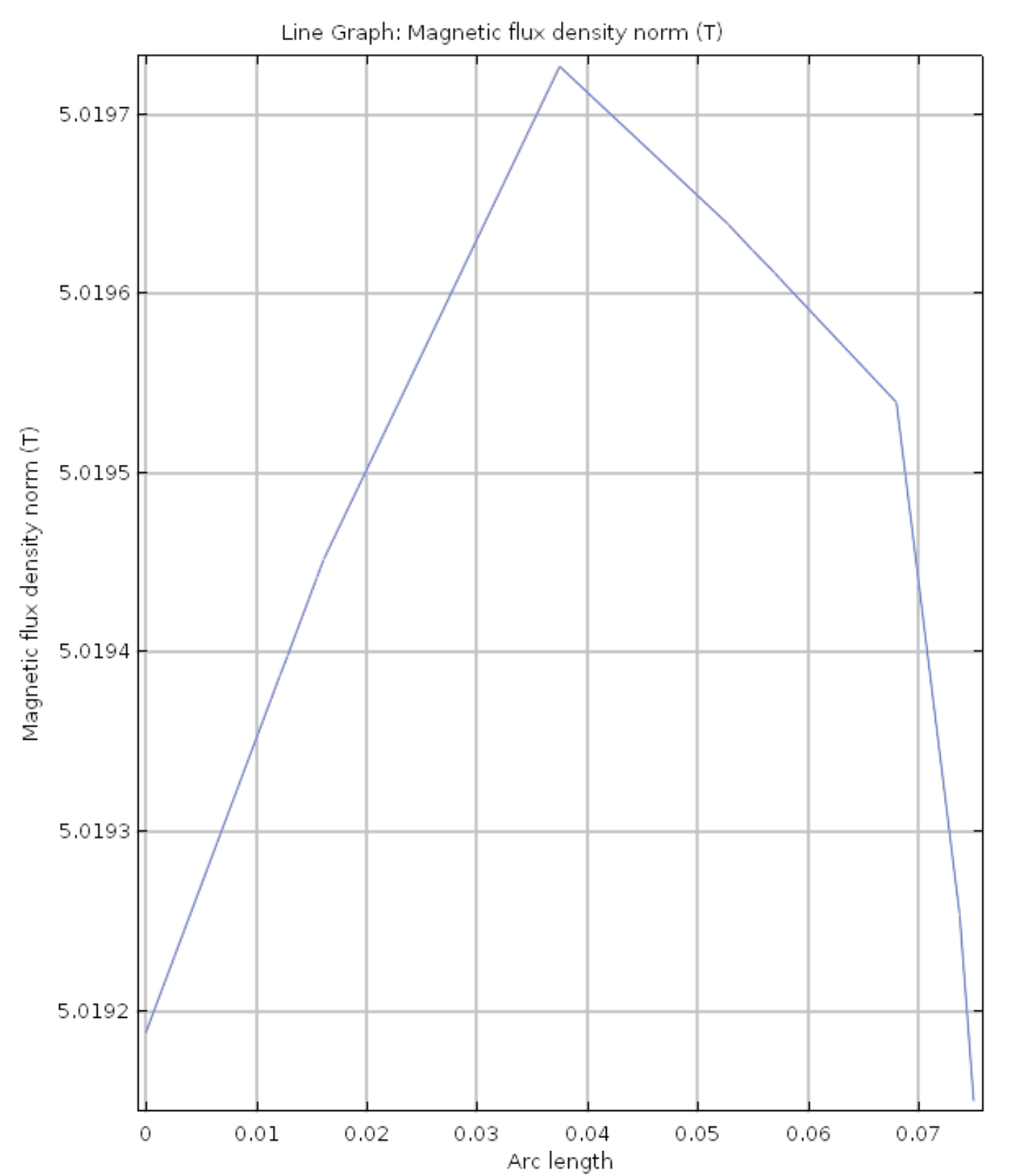

$Z=[-3.75 \mathrm{~cm} ; 3.75 \mathrm{~cm}], Y=0 \mathrm{~cm}$

Simulation

$B=[5.0192 \mathrm{~T} ; 5.0197 \mathrm{~T}]$
Measurement

$B=[5.0195 T ; 5.0199 \mathrm{~T}]$ 


\section{Magnetic Field in the Magnet}

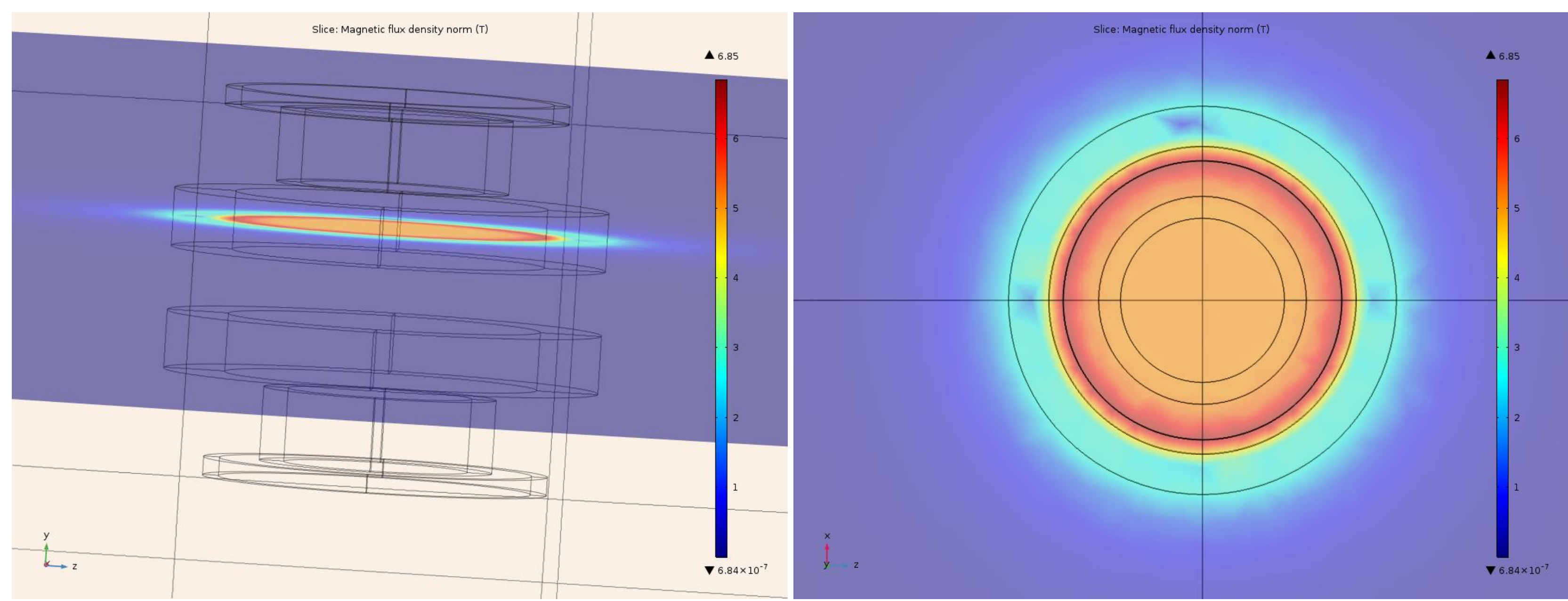

The magnetic field in the magnet is azimuthally symmetric (as expected) with the maximum field of $6.85 \mathrm{~T}$ at the inner surface of the coils. The Quench limit of the NbTi Superconductor for the $B=6.85 \mathrm{~T}$ is $\sim 6.35 \mathrm{~K}$ 


\section{Physics Processes}

- Heat Load

- Cooling processes

- Approximation Strategy 


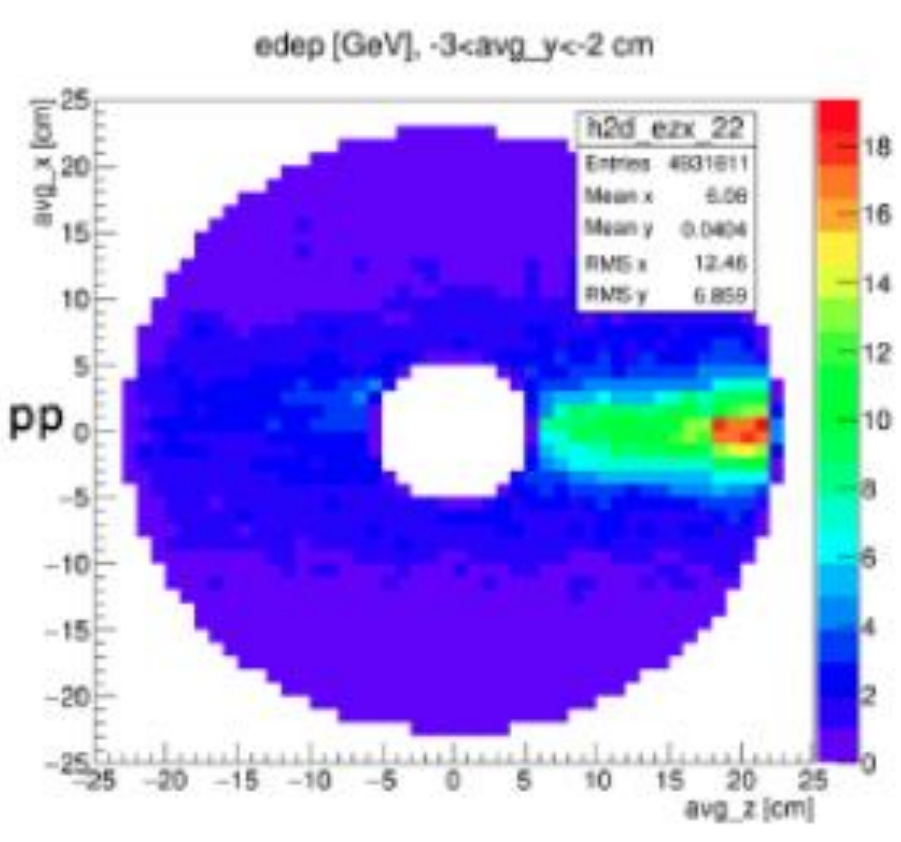

edep [GeV], -5<avg $y<-4 \mathrm{~cm}$

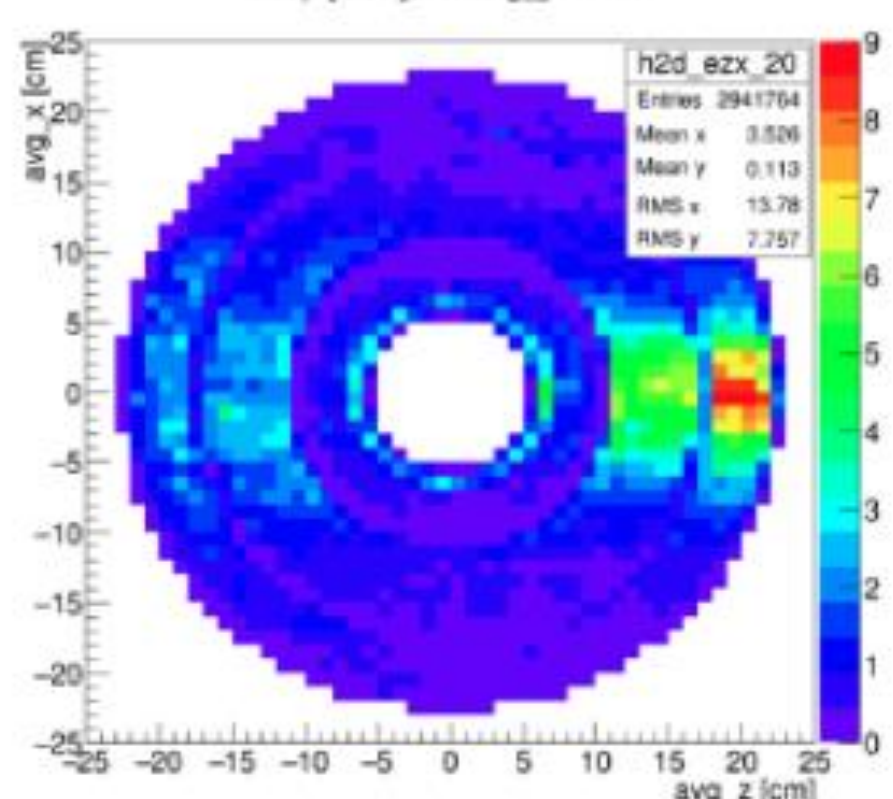

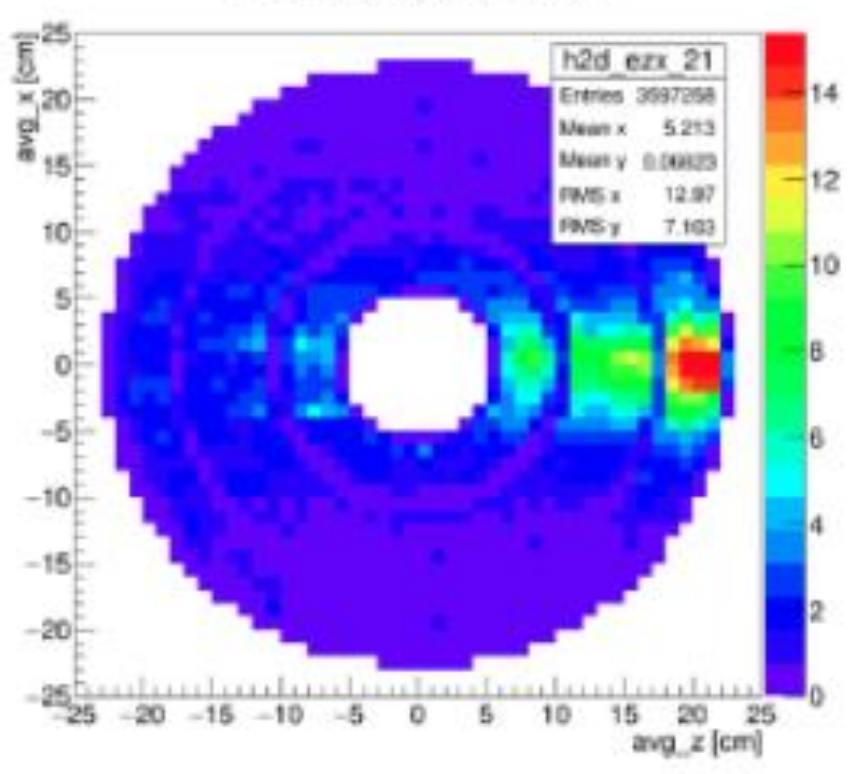

edep [GeV], 6eavg y<-5 cm

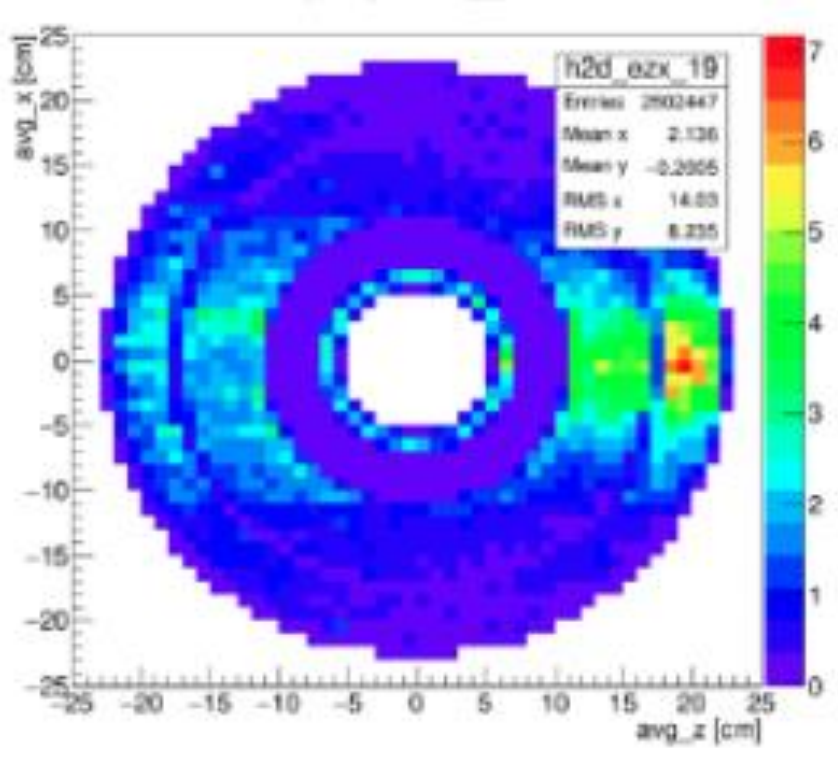

The heat load in the magnet are obtained from the GeantBased MC simulations

The hot spot in the downstream magnet comes from the beam-target interactions

The hot spot in the upstream magnet comes from the beam-collimator interactions 


\section{Cooling Processes}

Time-Structure of the Beam

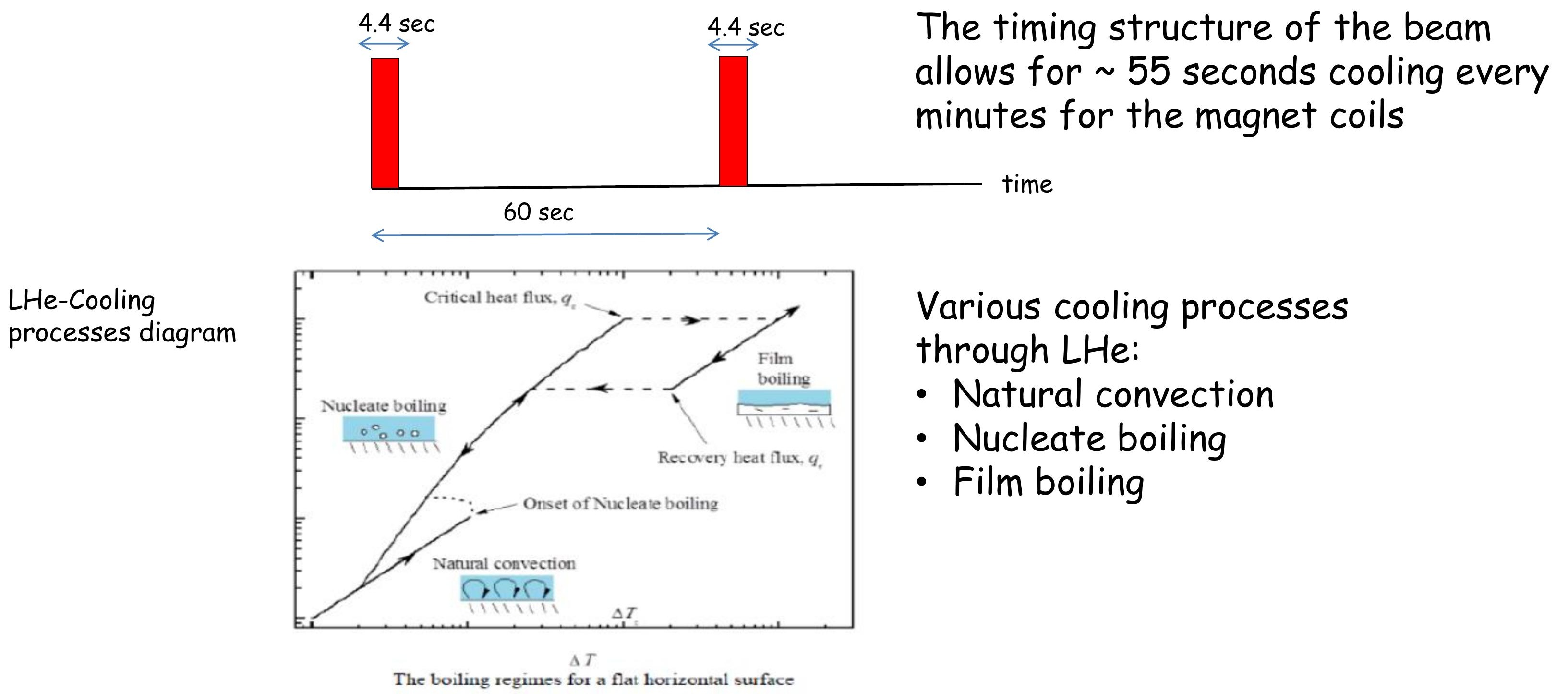




\section{Approximation Strategy}

Steady state is reached after $\sim 0.1 \mathrm{~s}$

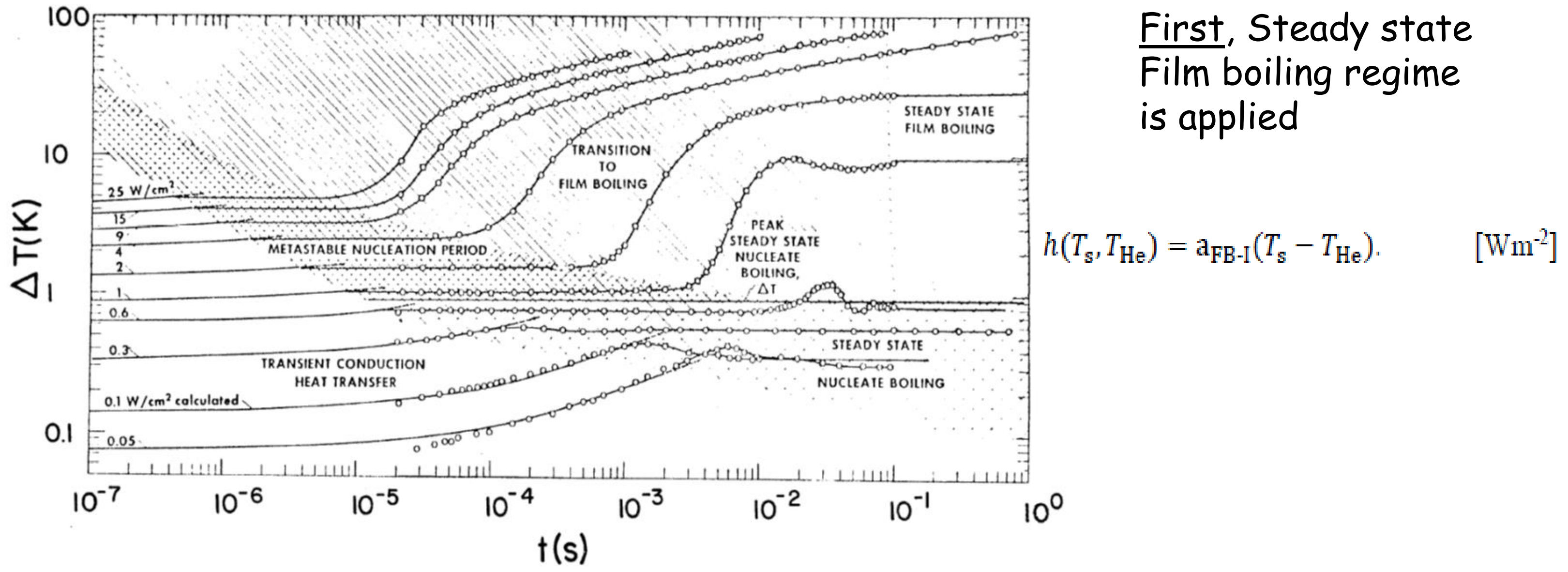

Various regimes of the heat transfer from solid to LHe 


\section{Approximation Strategy}

Second, we consider the superconducting magnet as a composite material with the effective thermal parameter

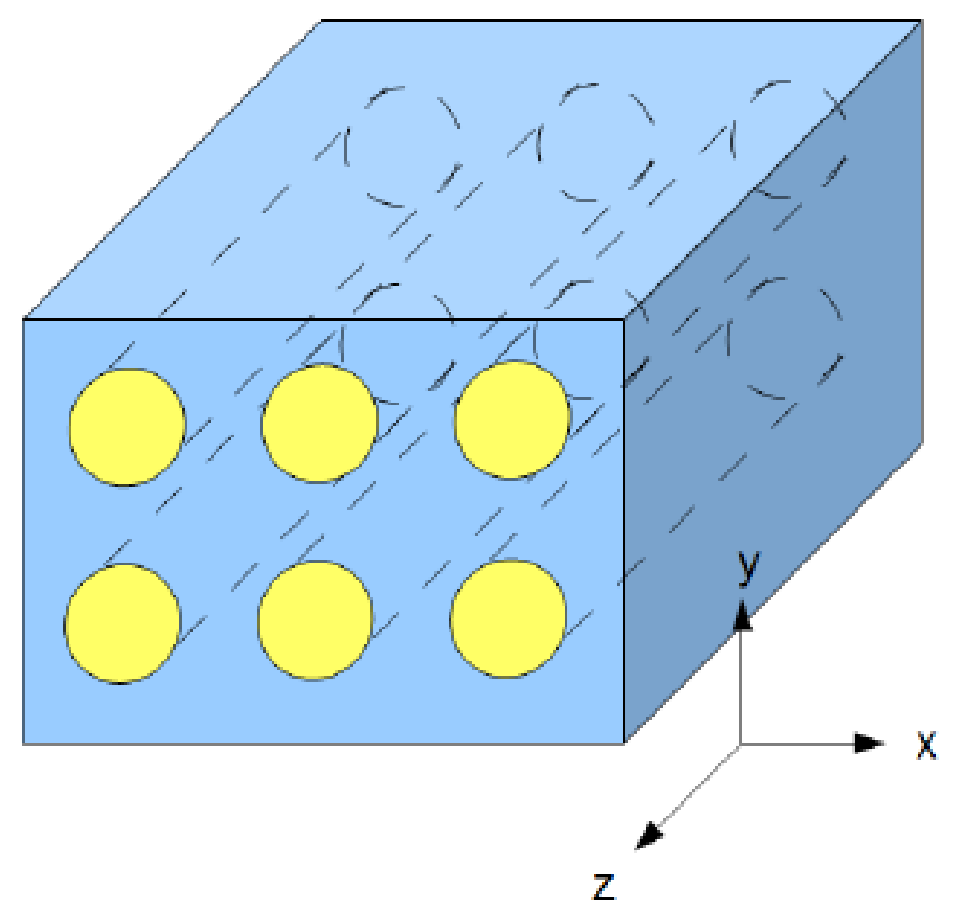

Rayleigh's formula for thermal conductivity

$\frac{k_{e f f}}{k_{m}}=1+\frac{3 \phi}{\left(\frac{k_{1}-2 k_{m}}{k_{1}-k_{m}}\right)-\phi+1.569\left(\frac{k_{1}-k_{m}}{3 k_{1}-4 k_{m}}\right) \phi^{\frac{10}{3}}+\ldots}$

Rayleigh's model consist of parallel cylinders embedded in a continuous matrix

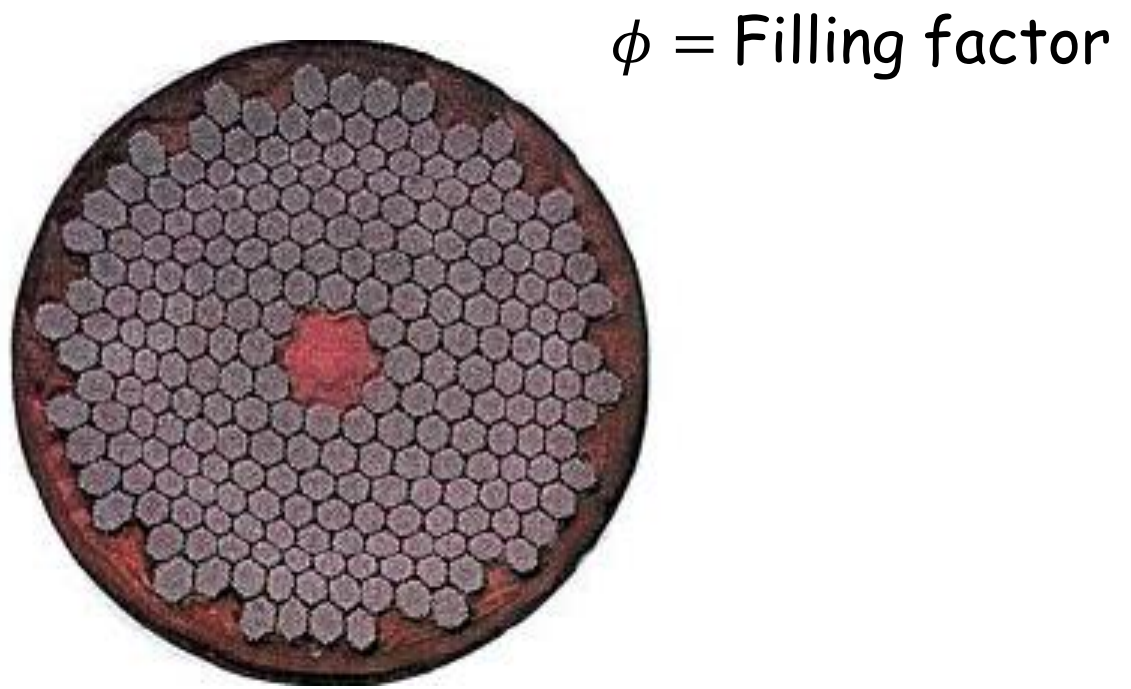




\section{Approximation Strategy}

Third, we parameterize some of the unknown properties by the effective surfaces that are in direct contact with the LHe:

- Parameter of the He void

- Insulation

- Former
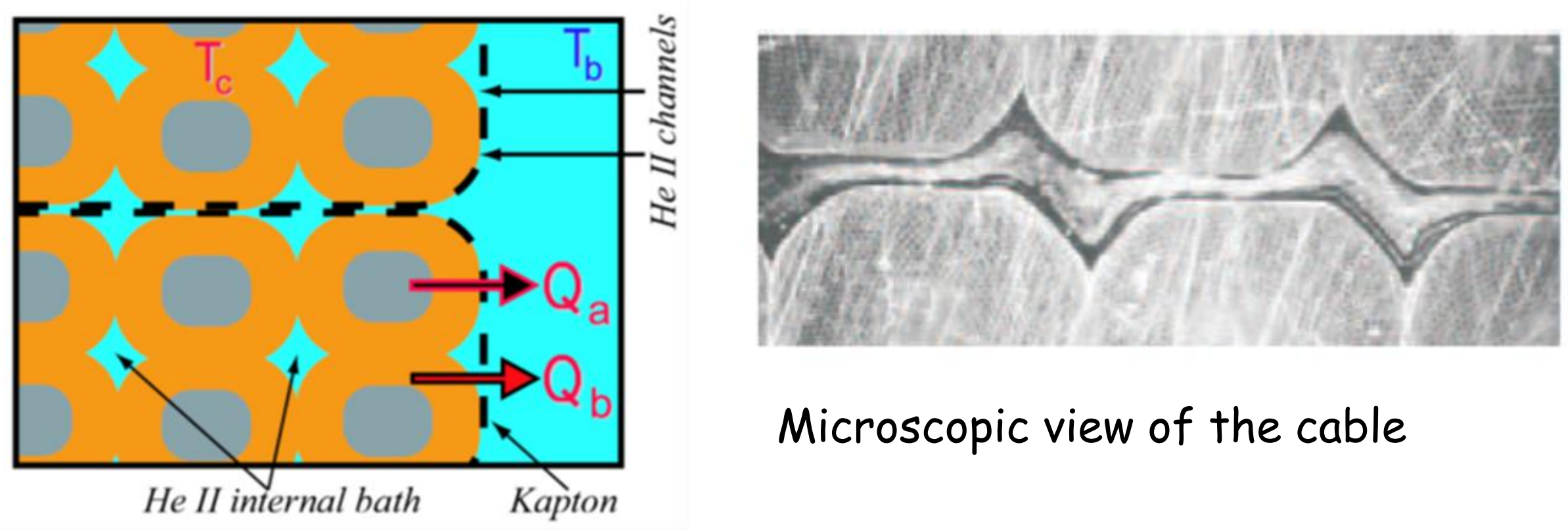

Microscopic view of the cable 


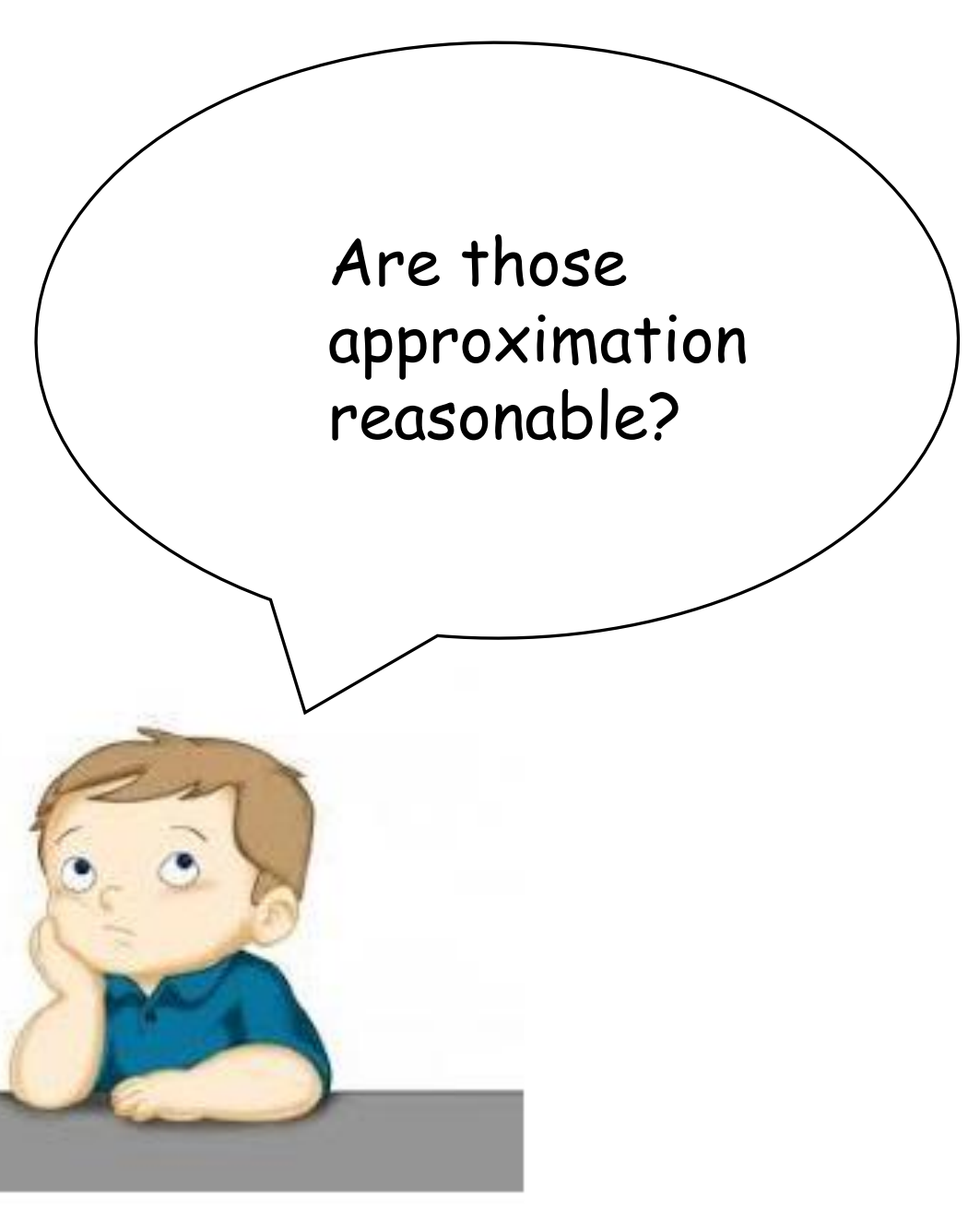

The time scale is large enough to take film boiling regime as an approximation

The film boiling heat transfer equation is linear $h\left(T_{\mathrm{s}}, T_{\mathrm{He}}\right)=\mathrm{a}_{\mathrm{FB}-\mathrm{I}}\left(T_{\mathrm{s}}-T_{\mathrm{He}}\right)$.

Where the coefficient is in $\mathrm{Wm}^{-2} \mathrm{~K}^{-1}$

Therefore the effective surface contact can be absorbed into this coefficient

We have quite large temperature margin (4K) since we operate in the normal phase of $\mathrm{He}$ (evaporation fridge)

Some systems that require to be operated in the superfluid He phase have temperature margin less than $1 \mathrm{~K}$ (even $\mathrm{mK}$ ) 


\section{Simulation Method}

Finite element analysis using COMSOL Multiphysics

\section{Input:}

- Volumetric heat source (Heat Map)

- Thermal properties of the material

- Heat transfer in solid and heat flux to the LHe

- Beam profile

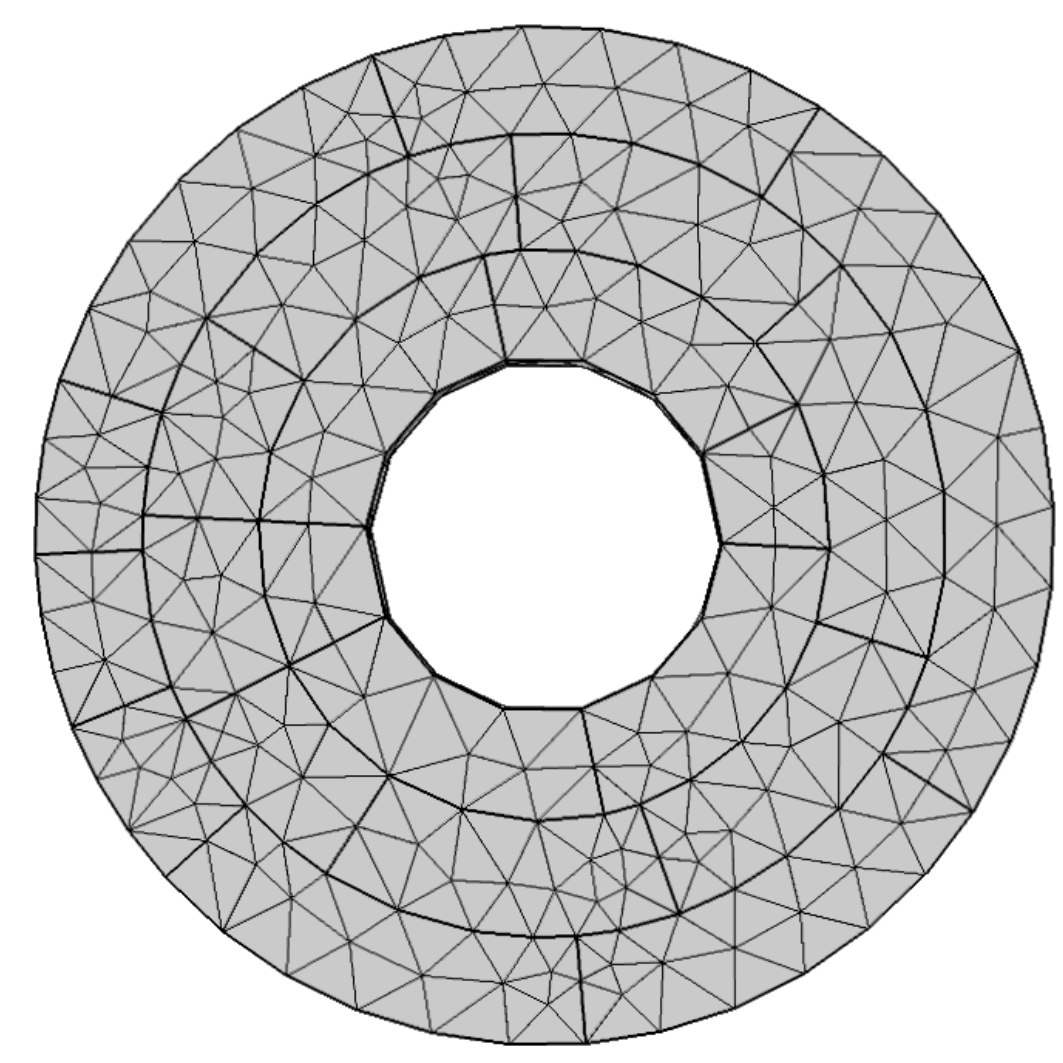

Proses: Discretized element for the Finite Element calculation 


\section{Results}

- BNL VS Spinquest

- Temperature profile T(x)

- Temperature profile $T \max (t)$ 


\section{Result}

SpinQuest VS BNL

SpinQuest

Time-Structure of the Beam

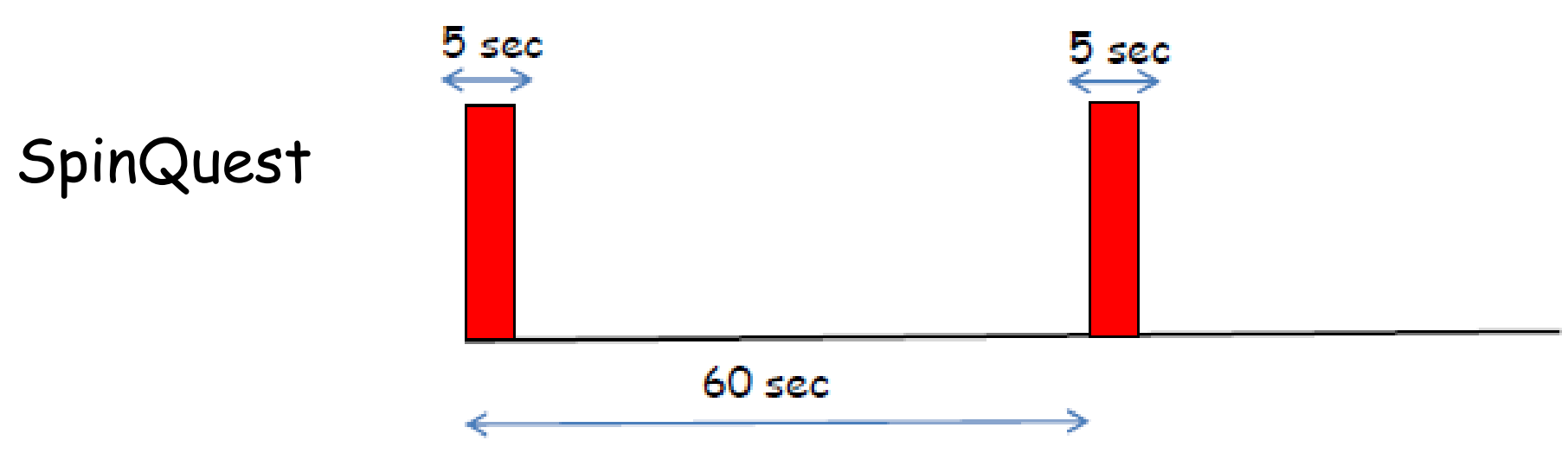

Time-Structure of the Beam

BNL

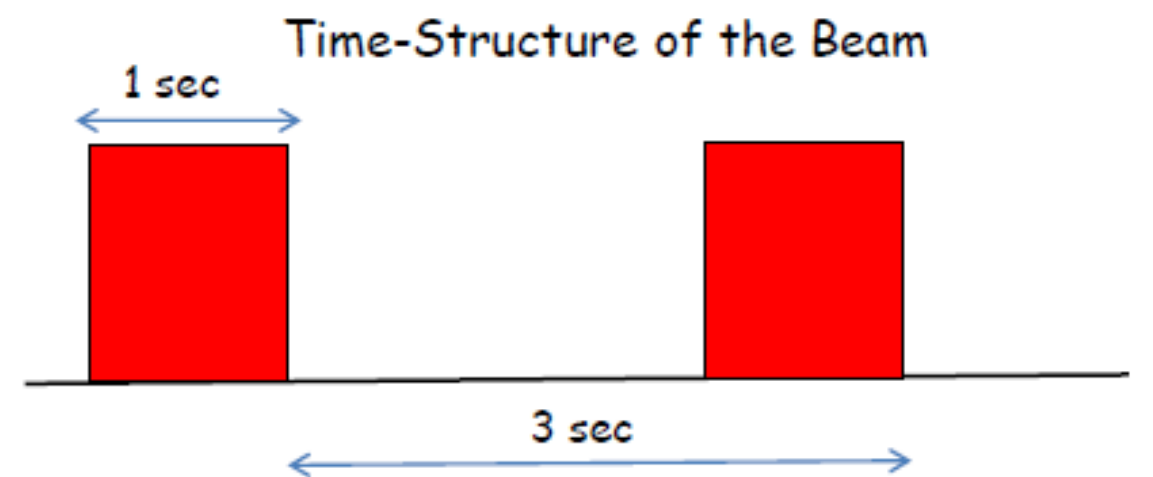

SpinQuest beam profile

\begin{tabular}{|c|c|}
\hline Energy & $120 \mathrm{GeV}$ \\
\hline Cycle Time & $60 \mathrm{~s}$ \\
\hline Spill Length & $4.4 \mathrm{~s}$ \\
\hline Beam Intensity & $1 \mathrm{e} 12$ \\
\hline
\end{tabular}

BNL beam profile

\begin{tabular}{|c|c|}
\hline Energy & $24 \mathrm{GeV}$ \\
\hline Cycle Time & 3 seconds \\
\hline Spill Length & 1 second \\
\hline Beam Intensity & $2 \times 10^{11}$ protons/pulse \\
\hline
\end{tabular}




\section{Results}

The temperature profile at $4.4 \mathrm{~s}$

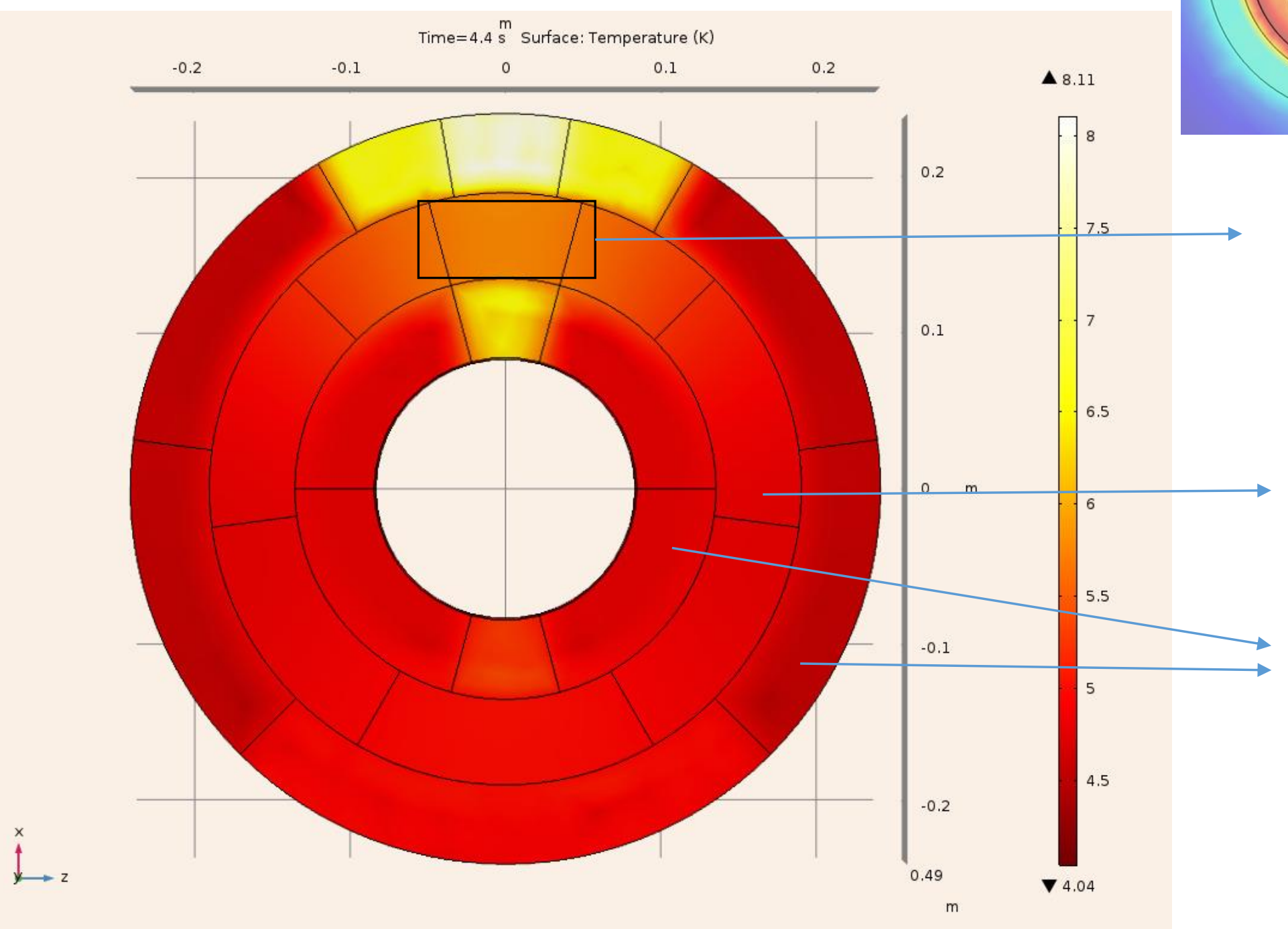

B-Profile of the magnet. The quench limit for the Bmax is $6.35 \mathrm{~T}$

The hot spot spread uniformly due to the thermal conductivity of the copper matrix

Magnet coil

Stainless-steel former 


\section{Results: SpinQuest}

The maximum temperature of the coil as a function of time $\sim 6.1 \mathrm{~K}$

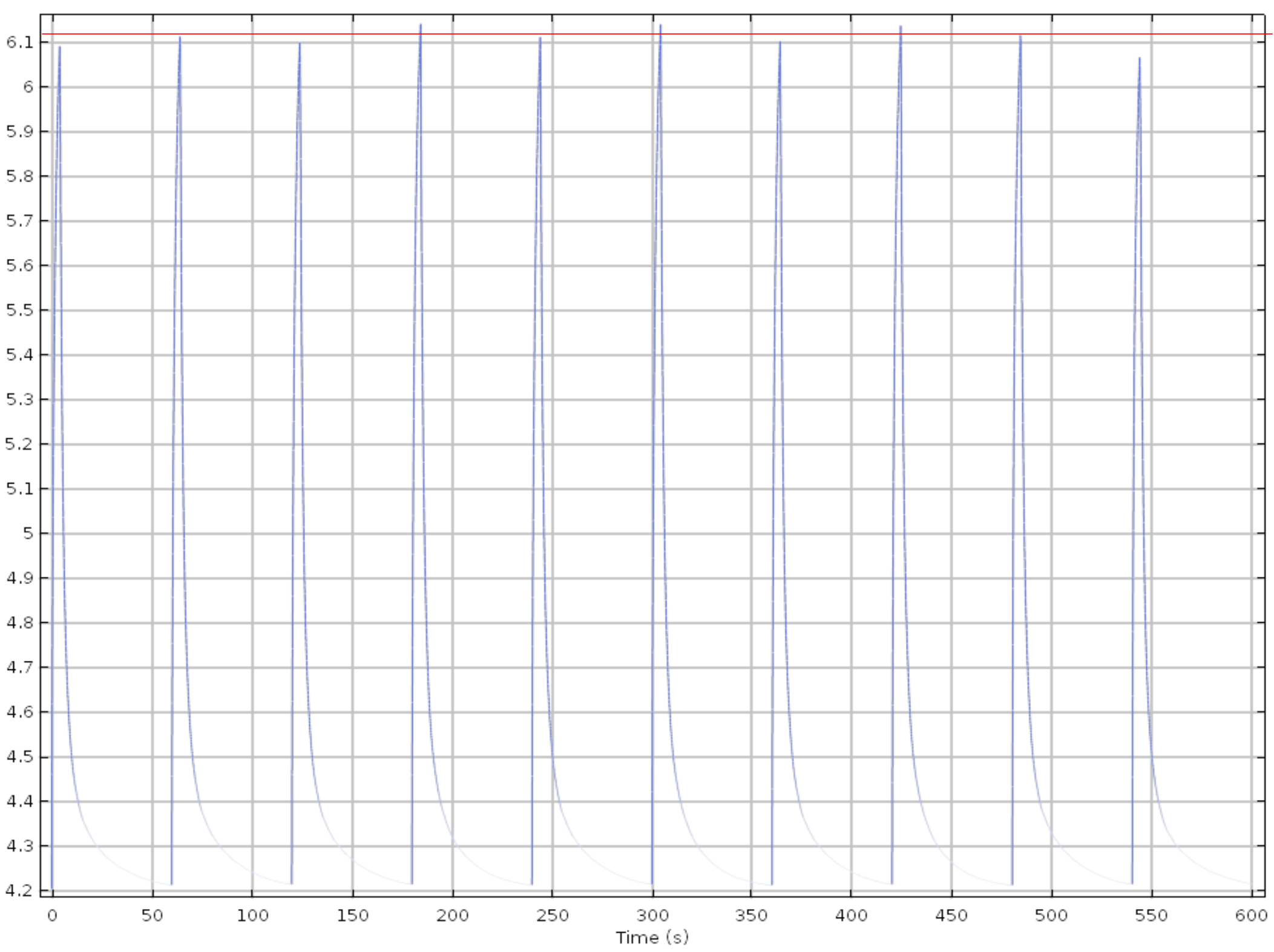

Maximum Temperature profile Tmax(t) for E1039:

- $120 \mathrm{GeV}$ proton

- 1 e12 proton/s

- NH3 Target

Conclusion: It is safe to run at 1 e12 proton/s but this intensity is considered as the upper limit (maximum) for the $\mathrm{NH} 3$ target 


\section{Results: BNL}

The maximum temperature of the coil as a function of time

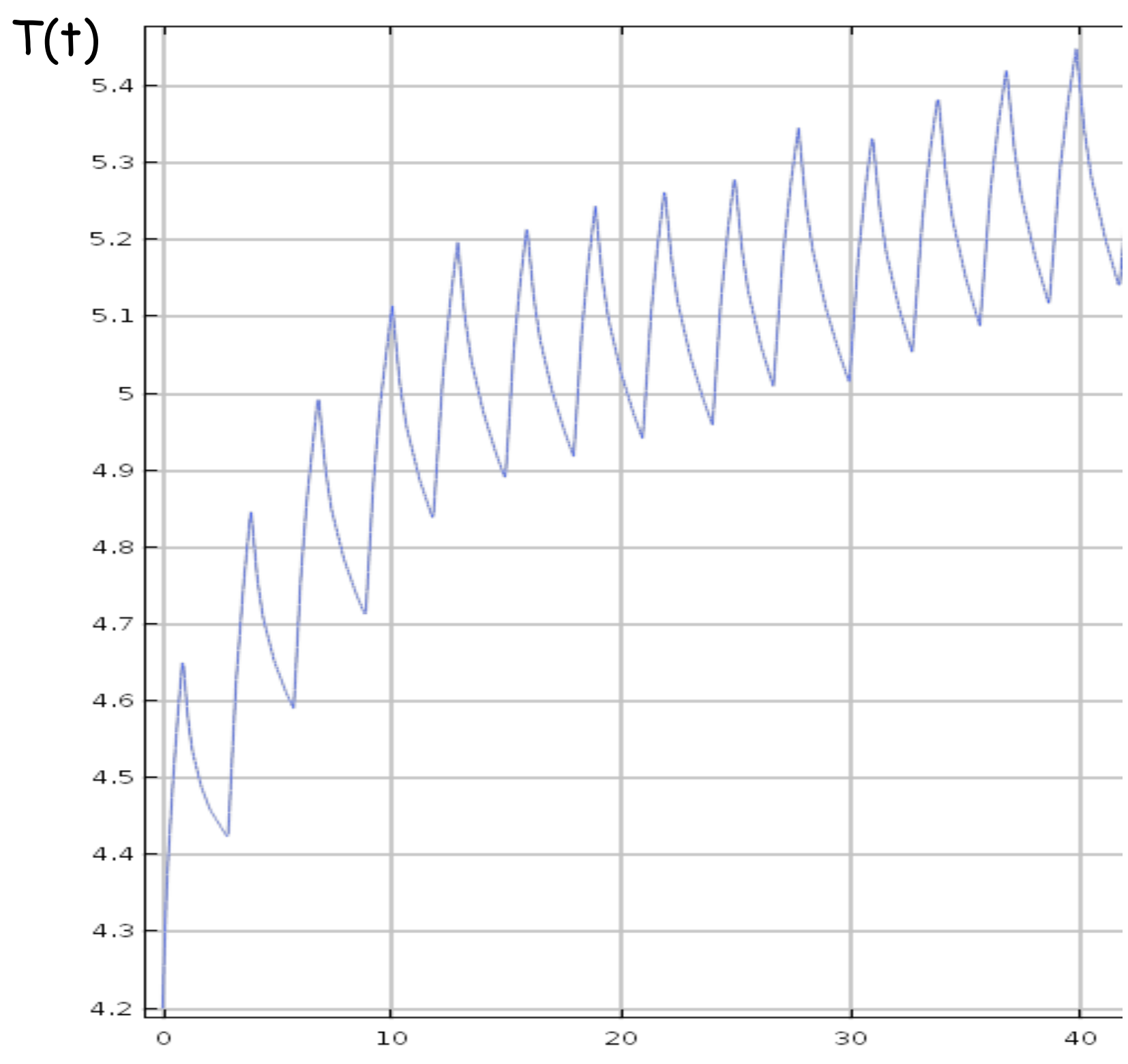

Maximum Temperature profile Tmax $(t)$ for BNL:

- $24 \mathrm{GeV}$ proton

- 2 e11 proton/s

- Teflon Target

Notes:

- The BNL magnet was quenched in this setup (Teflon target \& $2 e 11$ proton/s)

- The simulation results "indicate" quench $\rightarrow$ The heat is accumulated over time 


\section{Outlook: Quench commissioning plan}

- 8 Type-T Thermocouple sensors are installed in the surface of the stainless-steel former

- 4 sensors are installed upstream of the magnet and 4 sensors are installed downstream of the magnet

- The next 3 slides show the temperature prediction of the sensors for various beam intensities as indirect way to validate the simulation

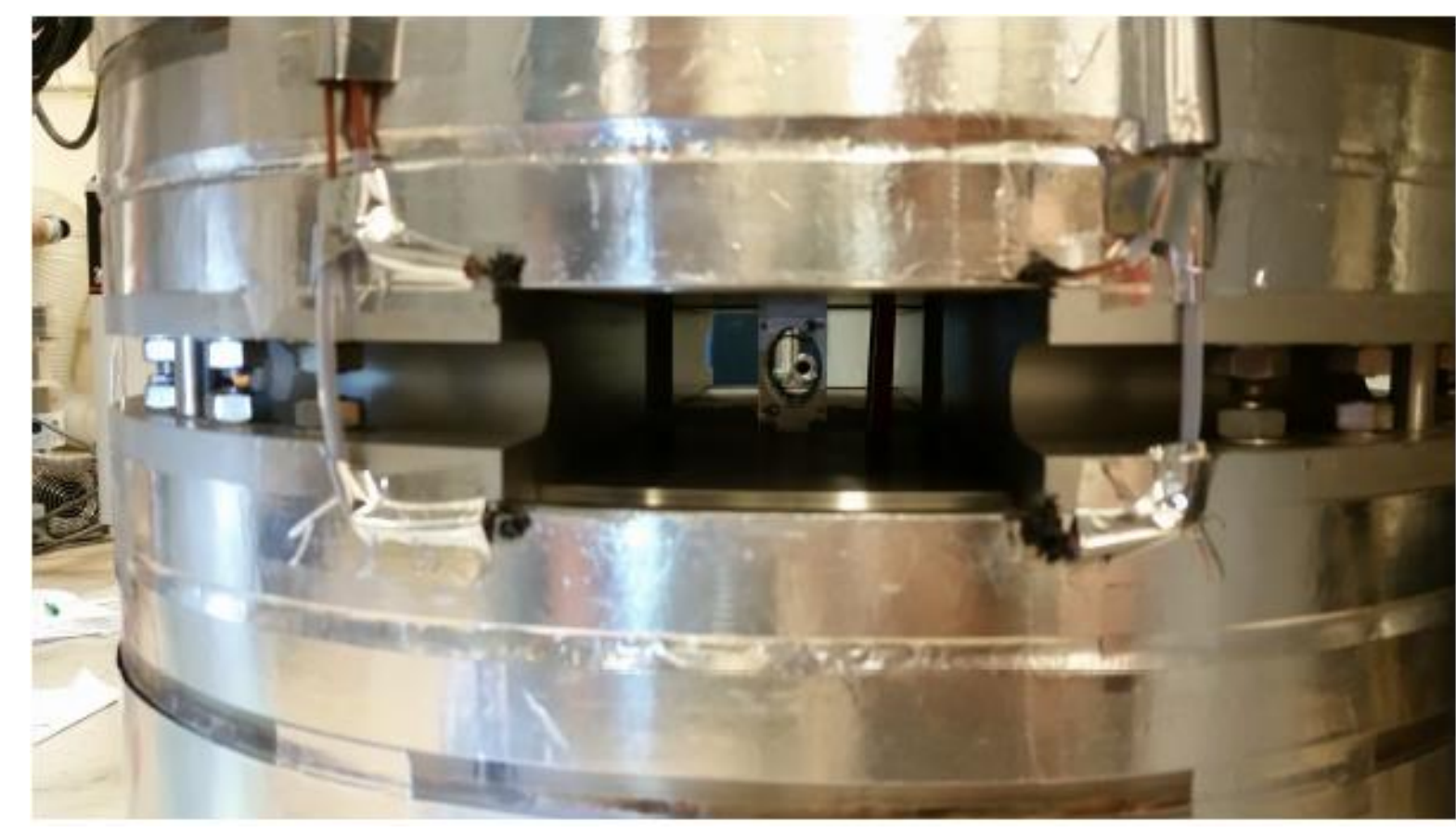

Type-T Thermocouple sensors are installed upstream/downstream of the magnet 


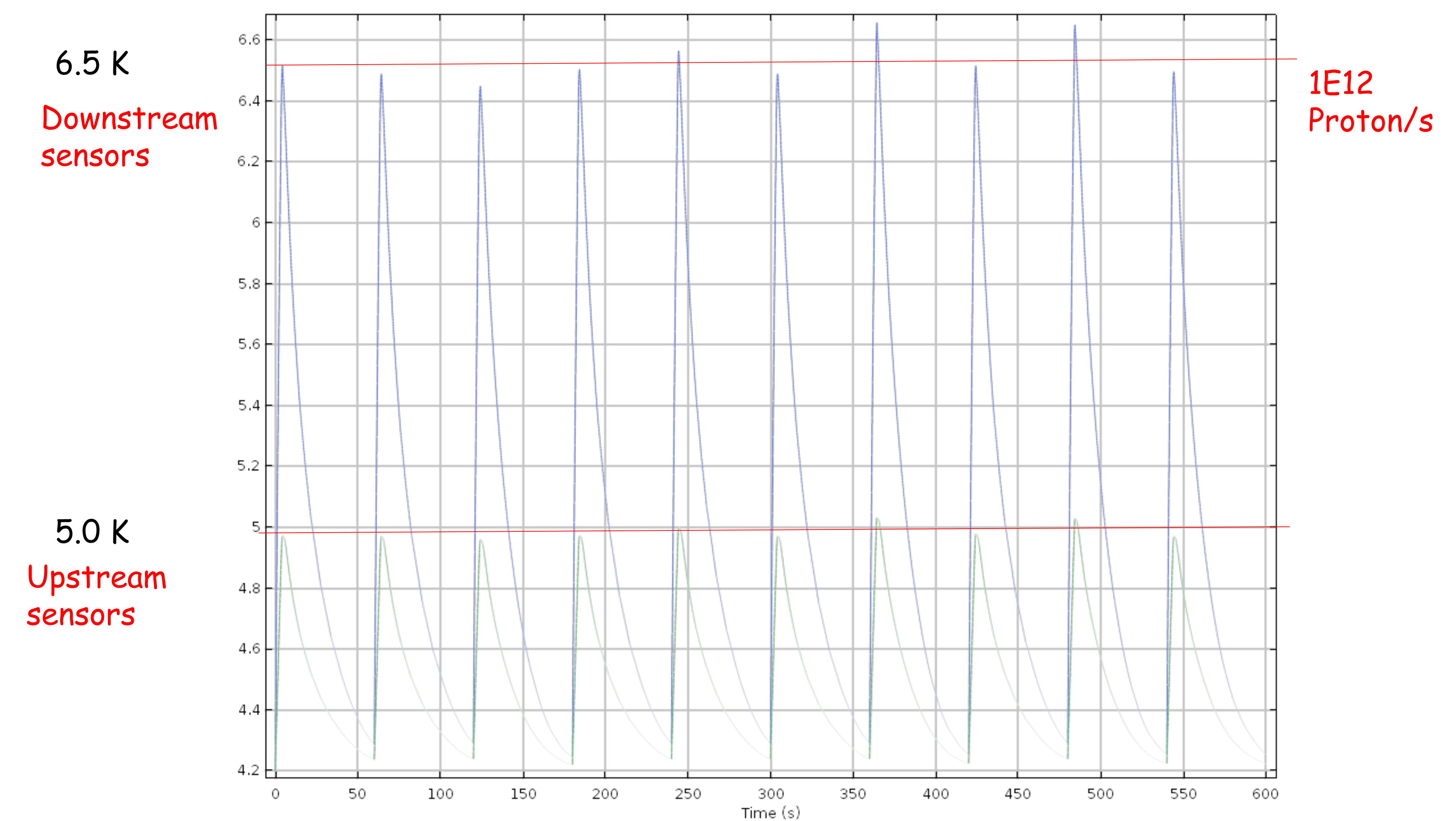


$5.5 \mathrm{~K}$

Downstream sensors

\section{$4.6 \mathrm{~K}$}

Upstream sensors
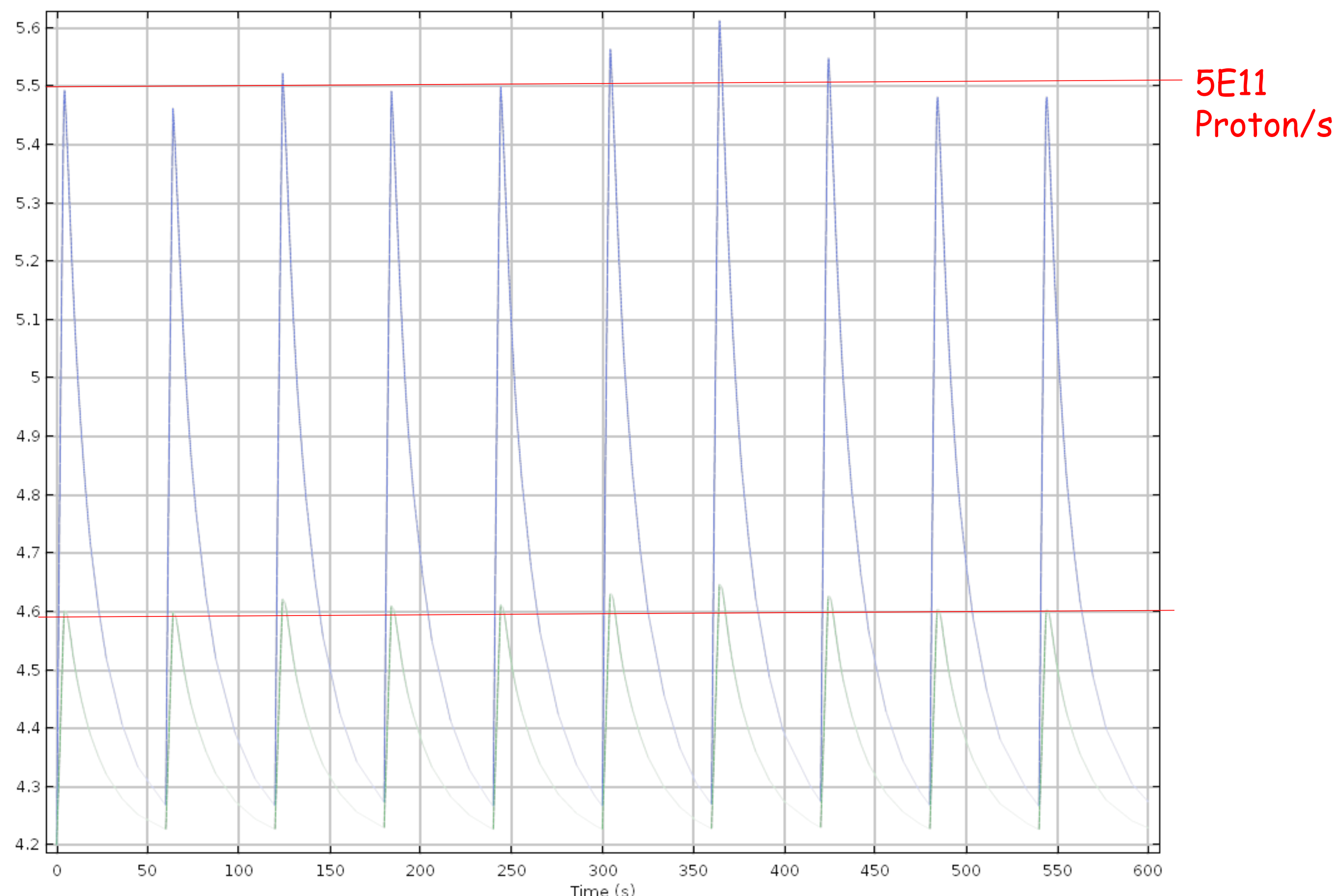


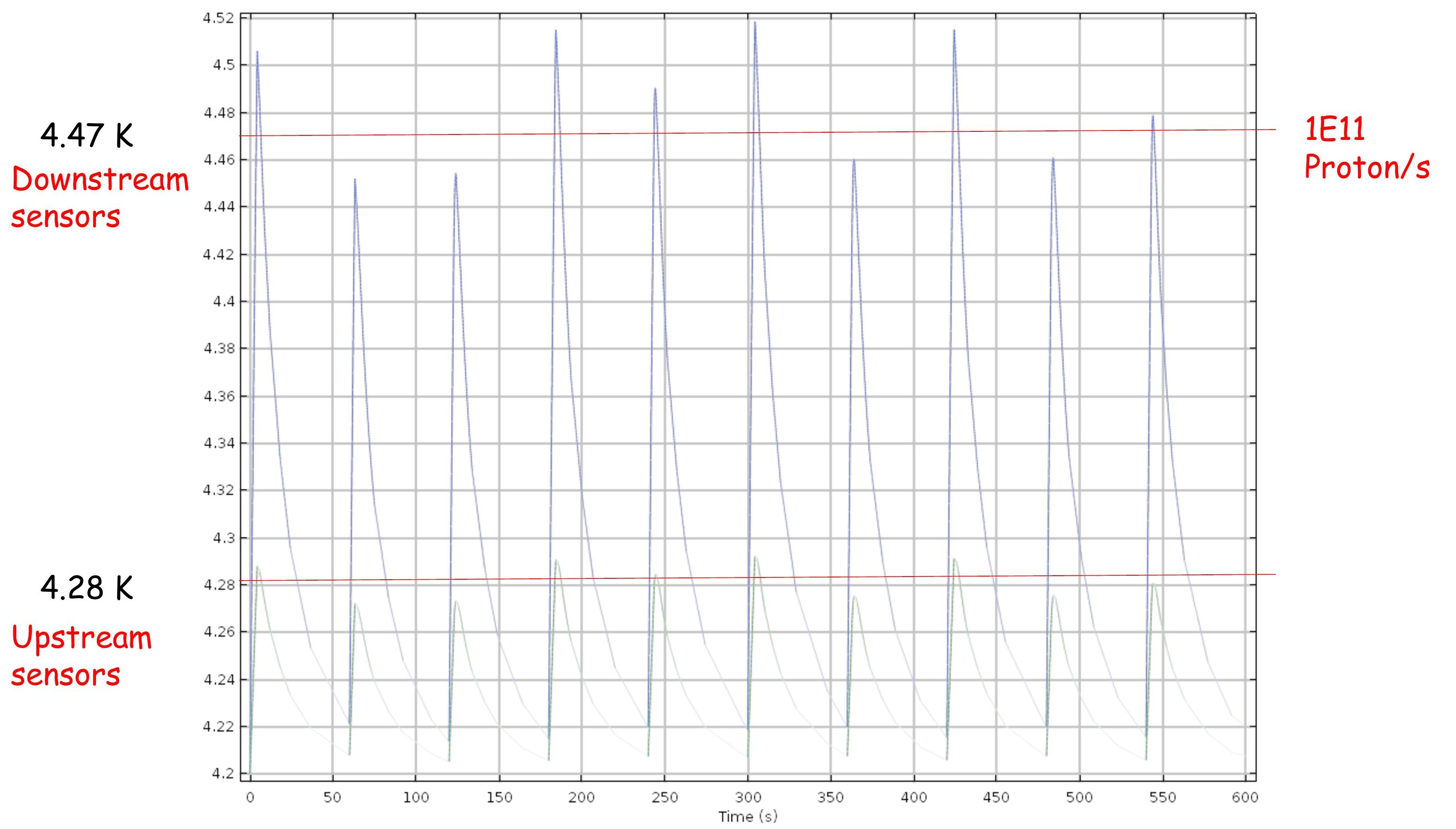


Thank You 


\section{Backup Slide}

Beam Stability Issue

- Intensity instability

- Beam drift 
The beam intensity "jump" in a very short period of time (ns)

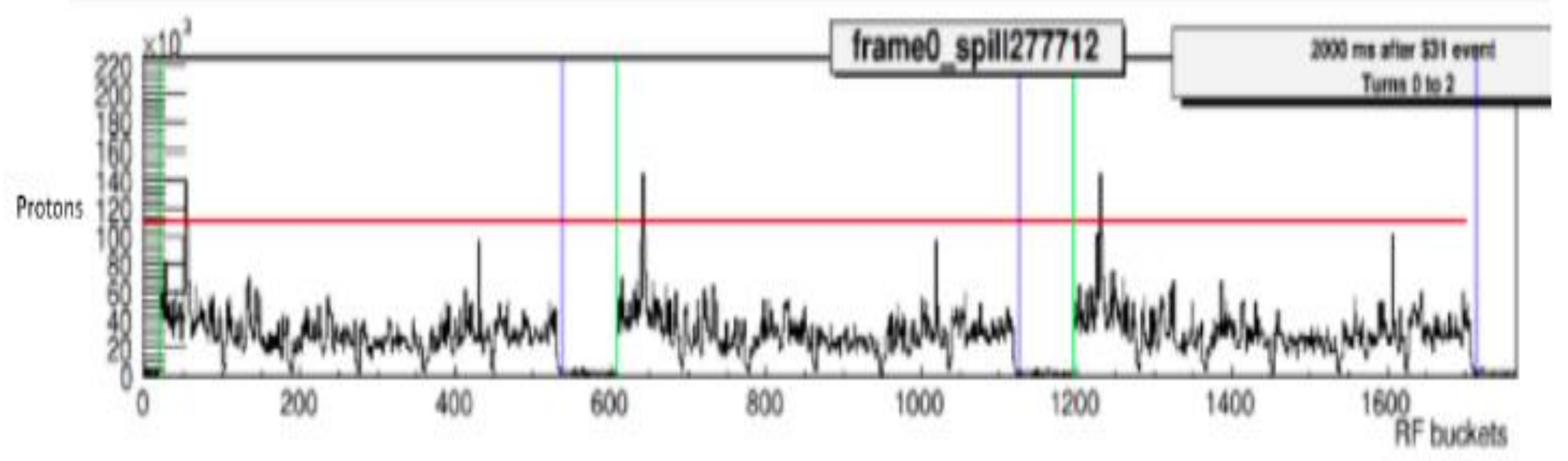

E906 temporal beam profile

Challenge: The simulation could not handle time scale of ns

Solution: Analytic calculation with some approximation 
$\frac{\partial}{\partial x}\left(k \frac{\partial T}{\partial x}\right)+\frac{\partial}{\partial y}\left(k \frac{\partial T}{\partial y}\right)+\frac{\partial}{\partial z}\left(k \frac{\partial T}{\partial z}\right)+\dot{q}=\rho c_{p} \frac{\partial T}{\partial t}$

Assumption for the upper limit of Temperature approximation: In a very short period of time, the Heat are localized $\rightarrow k=0$

If this assumption is correct, the difference between the calculation and real simulation should going smaller (match) as the time become smaller

\begin{tabular}{|l|l|l|l|l|}
\hline $\begin{array}{l}\text { "Jump" } \\
\text { intensity }\end{array}$ & $\begin{array}{l}\text { Duration } \\
\text { of the } \\
\text { jump }\end{array}$ & $\begin{array}{l}\text { Tmax } \\
\text { Comsol (K) }\end{array}$ & $\begin{array}{l}\text { Tmax } \\
\text { Calculation } \\
\text { (K) }\end{array}$ & Delta T \\
\hline 10 times & 0.2 & 7.3 & 10.2 & 2.87 \\
\hline 10 times & 0.15 & 7 & 9.05 & 2.05 \\
\hline 10 times & 0.125 & 6.7 & 8.44 & 1.74 \\
\hline 10 times & 0.1 & 6.3 & 7.78 & 1.48 \\
\hline
\end{tabular}


Simulation for $t=0.2 \mathrm{~s}$

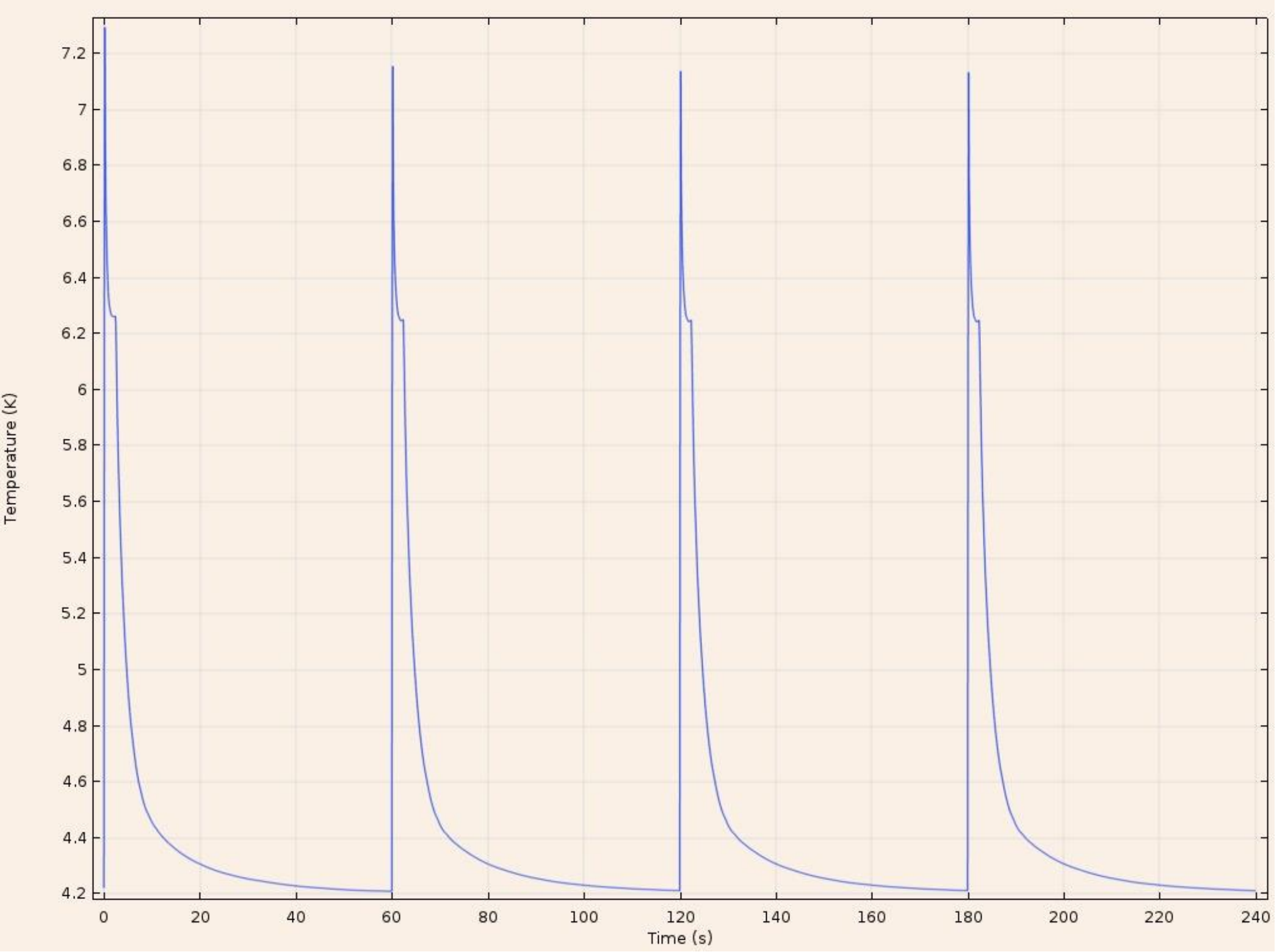

Calculation for $t=0.2 \mathrm{~s}$

$$
\begin{aligned}
T & =\sqrt{42.868 \times I \times t+17.64} \\
& =10.2 \mathrm{~K}
\end{aligned}
$$




\begin{tabular}{|l|l|l|l|l|}
\hline $\begin{array}{l}\text { "Jump" } \\
\text { intensity }\end{array}$ & $\begin{array}{l}\text { Duration } \\
\text { of the } \\
\text { jump }\end{array}$ & $\begin{array}{l}\text { Tmax } \\
\text { Comsol }(K)\end{array}$ & $\begin{array}{l}\text { Tmax } \\
\text { Calculation } \\
(K)\end{array}$ & Delta T \\
\hline 10 times & 0.2 & 7.3 & 10.2 & 2.87 \\
\hline 10 times & 0.15 & 7 & 9.05 & 2.05 \\
\hline 10 times & 0.125 & 6.7 & 8.44 & 1.74 \\
\hline 10 times & 0.1 & 6.3 & 7.78 & 1.48 \\
\hline
\end{tabular}

Since the Tmax calculation between simulation and calculation match as the time (duration of the jump) going smaller. We can trust the calculation. For the ns duration of the jump:

$$
\begin{aligned}
T & =\sqrt{42.868 \times I \times t+17.64} \\
& \sim 4.2 \mathrm{~K}
\end{aligned}
$$


Perfect beam alignment
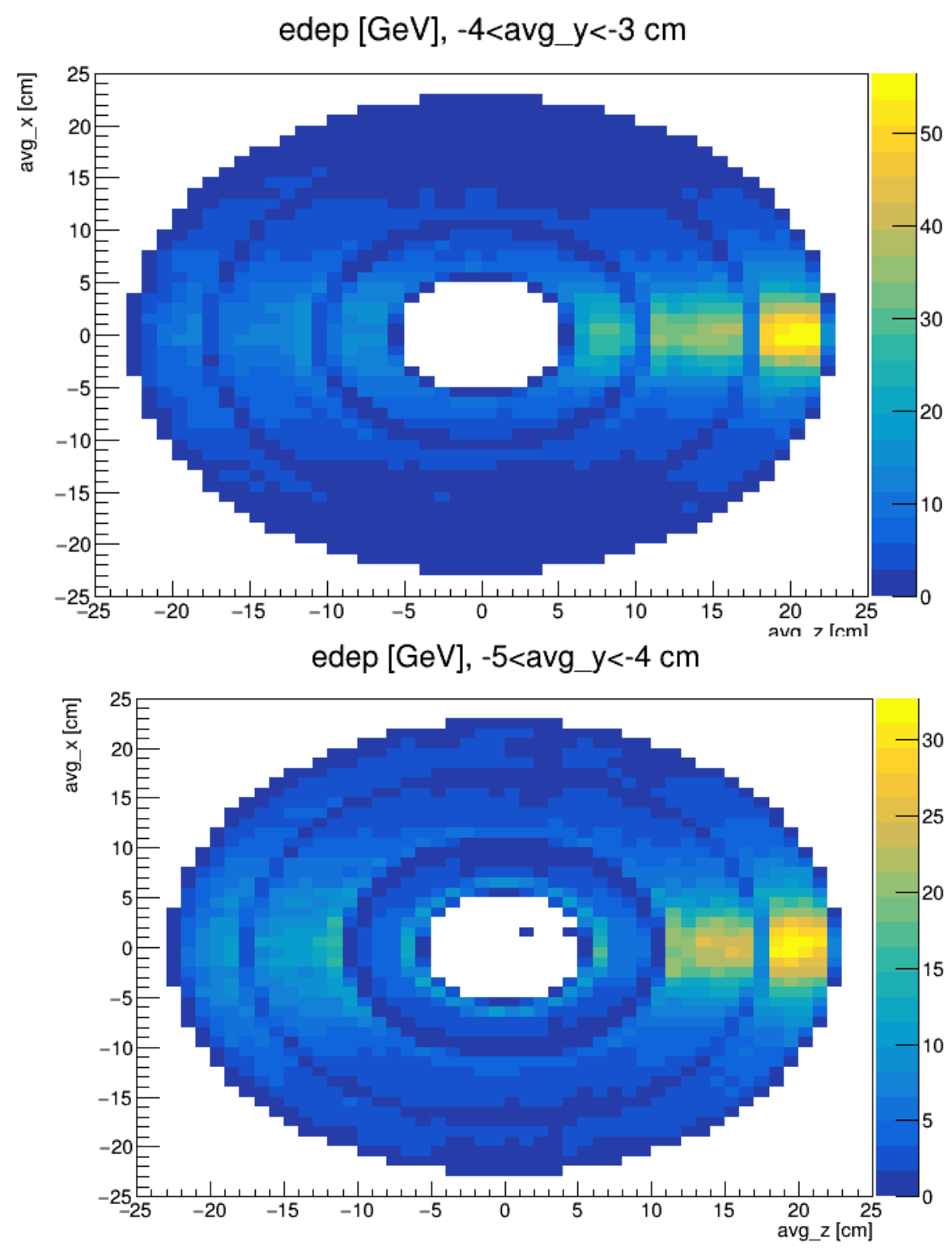

Beam drift or misalignment by $0.3 \mathrm{~cm}$

The energy deposited in the hot spot increase by 15\%

$0.1 \mathrm{~K}$ of temperature increase edep [GeV], $-4<$ avg $y<-3 \mathrm{~cm}$
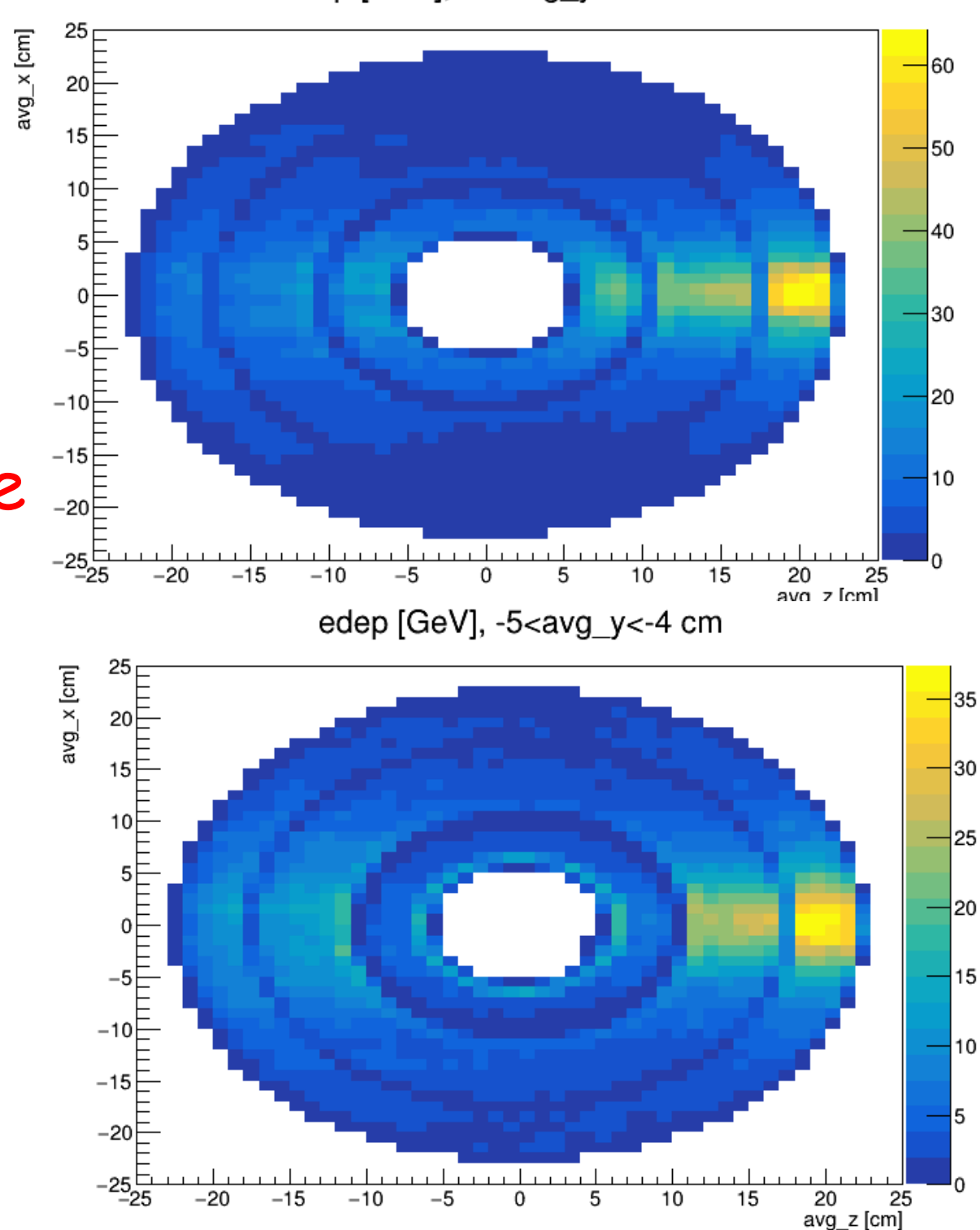
Temperature profile with a perfect

alignment showing the maximum

temperature of $6.1 \mathrm{~T}$

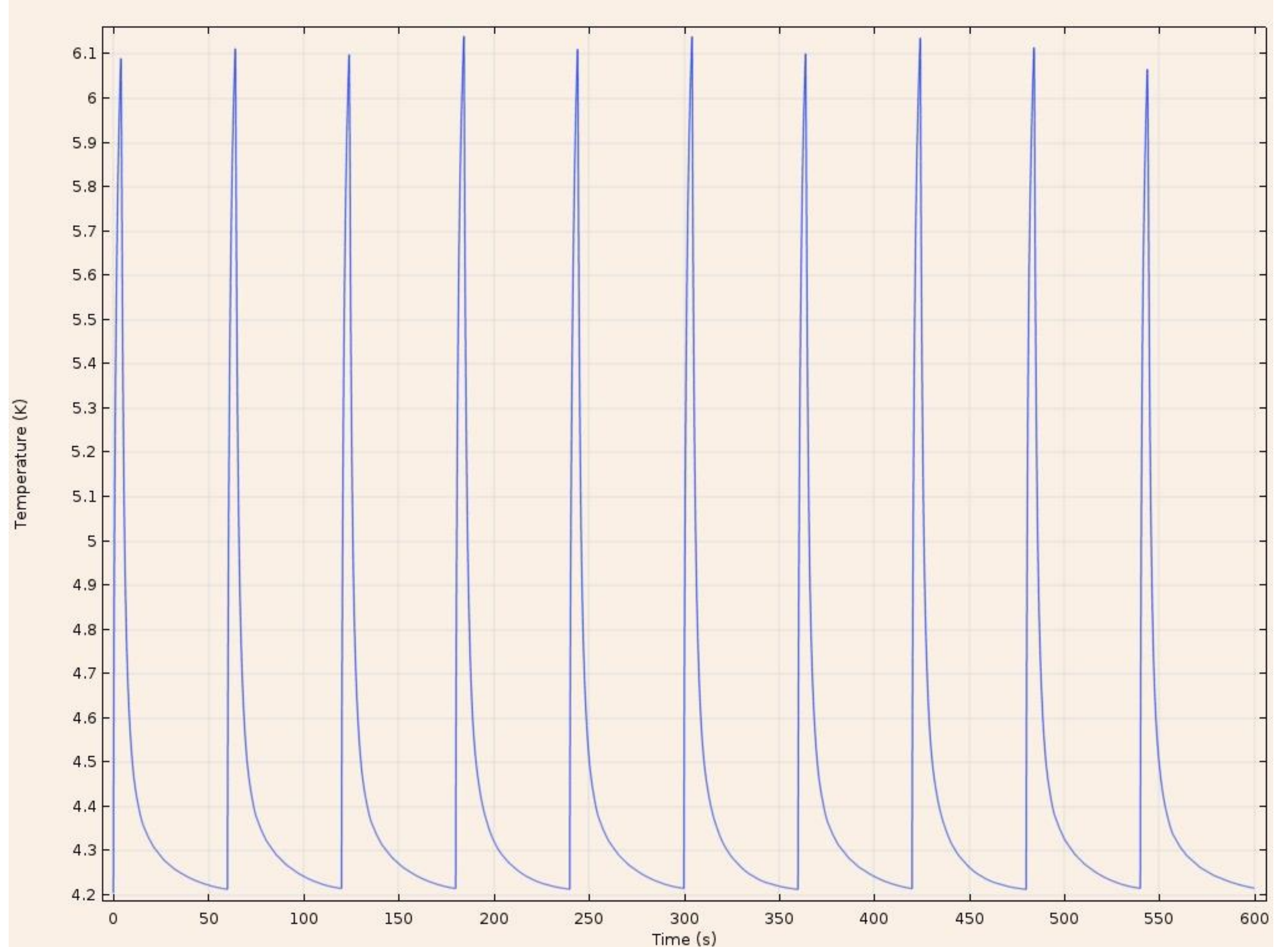


Temperature profile with the $0.3 \mathrm{~cm}$ of beam drift/misalignment showing the maximum temperature of $6.2 \mathrm{~T}$

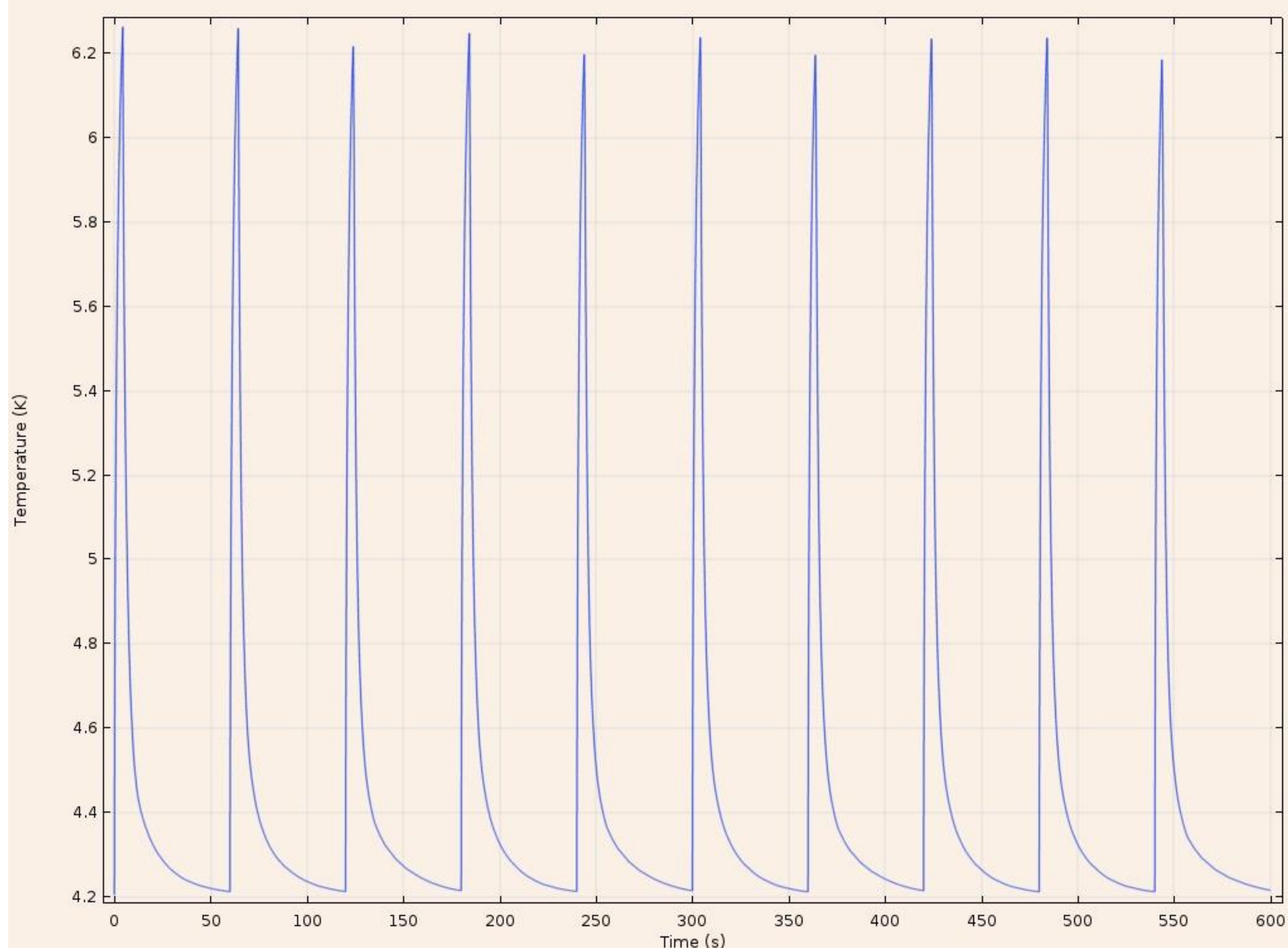




\section{Thank You}

